\title{
Invisibility and Cloaking: Origins, Present, and Future Perspectives
}

\author{
Romain Fleury, Francesco Monticone, and Andrea Alù* \\ Department of Electrical and Computer Engineering, University of Texas at Austin, \\ 1616 Guadalupe St. UTA 7.215, Austin, TX 78701, USA \\ (Received 14 April 2015; published 1 September 2015)
}

\begin{abstract}
The development of metamaterials, i.e., artificially structured materials that interact with waves in unconventional ways, has revolutionized our ability to manipulate the propagation of electromagnetic waves and their interaction with matter. One of the most exciting applications of metamaterial science is related to the possibility of totally suppressing the scattering of an object using an invisibility cloak. Here, we review the available methods to make an object undetectable to electromagnetic waves, and we highlight the outstanding challenges that need to be addressed in order to obtain a fully functional coating capable of suppressing the total scattering of an object. Our outlook discusses how, while passive linear cloaks are fundamentally limited in terms of bandwidth of operation and overall scattering suppression, active and/or nonlinear cloaks hold the promise to overcome, at least partially, some of these limitations.
\end{abstract}

DOI: 10.1103/PhysRevApplied.4.037001

\section{INTRODUCTION}

Common materials, formed by charged particles that interact with electromagnetic waves, are inherently detectable using electromagnetic radiation. We experience this every time we look at an object: the reason why our eyes can see an object is that it reflects or scatters a fraction of the ambient light hitting its surface, and our retina can detect a portion of this scattered wave. The same principle is used in radar systems, which detect the scattering of a radio-frequency signal impinging on an unknown object. In order to make an object completely invisible, it is therefore necessary to cancel any scattering from it or, in other words, the propagation of electromagnetic waves must be the same regardless of whether the object is present or not. This implies that we need to modify the interaction of the object with electromagnetic waves by pairing it with a suitable engineered system whose presence influences the natural scattering signature of the object, canceling it. This is the purpose of invisibility cloaks.

Depending on the targeted application, the requirements for invisibility cloaks are more or less stringent. In the case of invisibility to the human eye, for instance, the operational bandwidth should cover the visible spectrum, but since the human eye is insensitive to the phase or the polarization of light, it may not matter whether the wave phase or polarization is conserved via scattering, while the most important quantities to conserve are the direction of propagation, frequency, and intensity. In other applications, for which the direction of the incident illumination is known, scattering cancellation for all angles is not required, which may simplify the cloak design. Some other

* Corresponding author. alu@mail.utexas.edu applications do not require a large bandwidth; for instance, cloaking receiving antennas requires cloaks with the same bandwidth as the antenna. In any case, the most important metrics for cloaks are (i) the amount of scattering reduction induced by the cloak and its angular dependence (scattering pattern) and (ii) the bandwidth of operation, i.e., the frequency band over which the object visibility is reduced to the point that common sensors cannot detect it.

Over the last century, problems related to cloaking have been investigated by the scientific community, such as the concept of invisible particles [1,2], nonradiating source distributions [3-7], low-scattering antennas [8-10], and the nonuniqueness of the inverse-scattering or tomography problem [11-14]. These studies suggest that cloaking may be theoretically possible, as they all describe the possibility of having polarized bodies that do not scatter. However, we had to wait for the development of the field of metamaterials over the past 15 years to see a significant breakthrough in the science and technology of invisibility. These structured materials are tailored to reach on-demand properties by putting together individually engineered scatterers that act as artificial atoms, resulting in an engineered propagation medium that interacts with waves in anomalous ways, achieving a degree of wave control that is impossible with natural materials [15-25]. Metamaterials have been associated with exotic phenomena, such as negative refraction [26-30], spatial localization and subwavelength focusing [27], spontaneous emission control [31-34], and a plethora of novel tunneling and waverouting effects [35-42]. Because of their superior wavemanipulation capabilities, metamaterials have made invisibility one step closer to reality.

This paper aims at analyzing the state of research in the field of invisibility and cloaking, and drawing perspectives on its future. In the first part, we review the most popular 
available methods to cancel the electromagnetic scattering from an object. We identify the main scientific challenges associated with designing and building a functional invisibility cloak, and highlight what remains to be done to transition these proof-of-concept prototypes to practical devices. In the second part, we build upon our analysis of the literature to show that passive linear cloaks are fundamentally limited in their ability to manipulate the scattering of an object over large bandwidths. We discuss how any scatterer is forced by causality, passivity, time independence, and linearity to abide by global bounds that limit the applicability of conventional passive cloaking schemes. The use of active and/or nonlinear cloaks, which do not abide by the above-mentioned bounds, is discussed in the third and final part of this article. We discuss promising examples that enable cloaking over broader bandwidth using active schemes. We conclude with an outlook on the future of this subject, which shows exciting perspectives involving active and nonlinear metamaterials and metasurfaces.

\section{CONVENTIONAL CLOAKING METHODS}

In order to set a basis for our analysis and vision, we now analyze the main methods to induce invisibility of a given object to electromagnetic waves using metamaterials. We aim at drawing a clearer picture of the principal challenges associated with passive cloaking techniques that have been developed over the past decade.

\section{A. Spherical cloaking based on transformation electrodynamics}

Transformation-based cloaking is a cloaking design method that uses the concept of transformation electrodynamics [43-51] to control the flow of electromagnetic energy using coordinate transformations that deform the coordinate grid of space, as in Fig. 1[52]. The idea starts by considering a ray of light propagating along a line in free space, as in Fig. 1(a). If we imagine that we have the ability to bend and stretch at will the coordinate grid of space, then, according to the Fermat principle, we can completely modify the path of the rays and redirect them, as shown in Fig. 1(b). Because of the form invariance of Maxwell's equation under a coordinate transformation, it is possible to interpret the
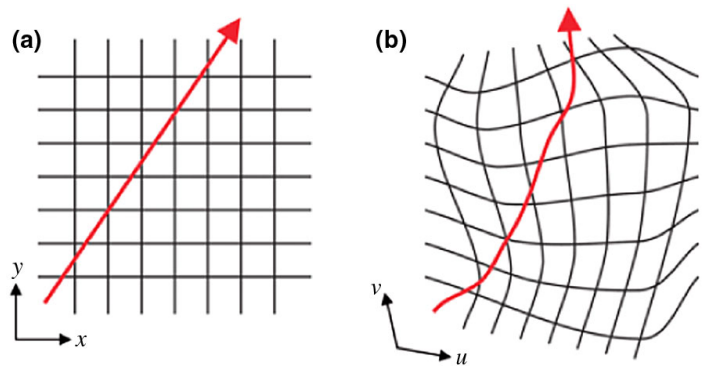

FIG. 1. Coordinate transformations can be used to manipulate the path of light. (C)2006 AAAS. Reprinted with permission, Ref. [52].
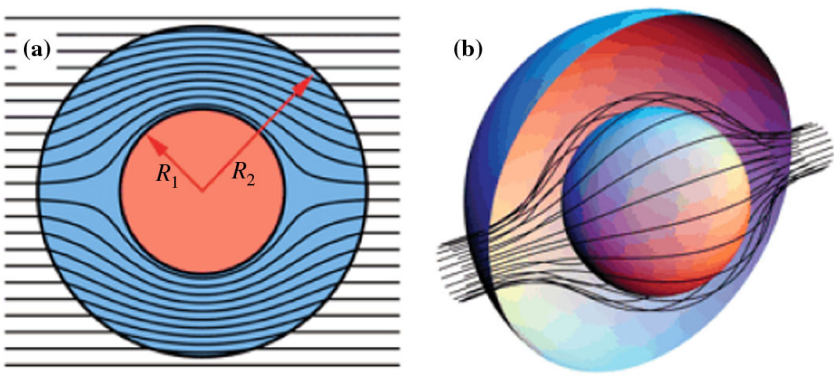

FIG. 2. Transformation-based spherical cloaking. (C)2006 AAAS. Reprinted with permission, Ref. [52].

propagation of light in the transformed coordinate system as the propagation in the untransformed system, but filled with a medium containing specific inhomogeneous and anisotropic permittivity and permeability distributions. According to transformation electrodynamics, if we could build a medium with such constitutive parameters, we would force light to follow the intended curved coordinates. Metamaterials, which enable full local control over their constitutive parameters, may represent the ideal platform to implement transformation optics in actual devices.

The possibility to apply transformation electrodynamics to the cloaking problem is well established and documented in the literature [52-69]. To do so, all one needs is to find a coordinate transformation that would redirect the flow of energy around a spherical region of space, in which the object to be concealed is placed. Transformation-electrodynamics equations can then automatically provide the required cloak design. A possible transformation is one that takes a spherical region of radius $R_{2}$ and maps its internal spherical volume into a spherical shell of internal radius $R_{1}$ and external radius $R_{2}$. The result of the transformation is basically to map free space into a curved space with a hole in it [Fig. 2(a)]. After mimicking this transformation using metamaterials, we would obtain a region of the actual physical space that is completely isolated from electromagnetic waves, in which we can hide an arbitrary object. Since the object cannot be reached by the electromagnetic energy, it cannot be polarized, and therefore cannot scatter. By design, the energy flow simply goes around the cloaked region and continues its way as if nothing were there [Fig. 2(b)].

For instance, using the radial transformation $r^{\prime}=R_{1}+r\left(R_{2}-R_{1}\right) / R_{1}$, we obtain the following constitutive tensor parameters for the cloak in the region $R_{1}<r<R_{2}$ [52]:

$$
\begin{aligned}
\varepsilon_{r r} & =\mu_{r r}=\frac{R_{2}}{R_{2}-R_{1}} \frac{\left(r-R_{1}\right)^{2}}{r^{2}}, \\
\varepsilon_{\theta \theta}=\mu_{\theta \theta} & =\frac{R_{2}}{R_{2}-R_{1}}, \\
\varepsilon_{\phi \phi} & =\mu_{\phi \phi}=\frac{R_{2}}{R_{2}-R_{1}} .
\end{aligned}
$$



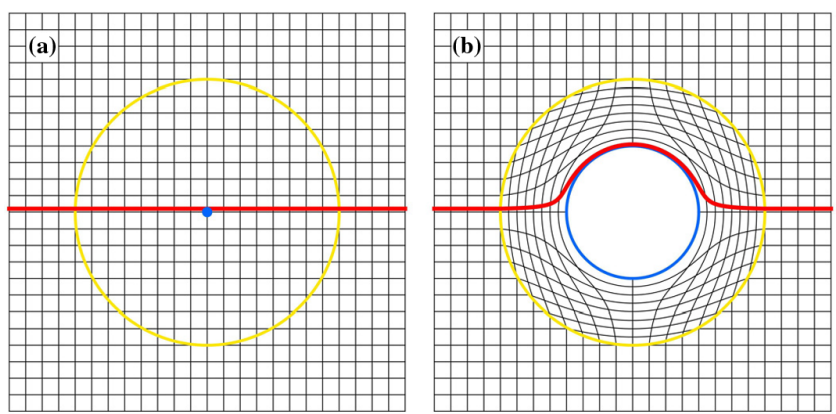

FIG. 3. Differences in path length in free space (a) and within the cloak (b) imply superluminal propagation. (C)2011 IOP Publishing. Reprinted with permission, Ref. [52].

From Eq. (1), we see that the magnetic and electric response for the cloak must be identical. This is to guarantee impedance matching to the background in the cloaking shell, ensuring no reflection and no scattering from the cloak itself. This also guarantees that the scattering response is not dependent on the polarization of the incident field, making the object invisible regardless of the illumination polarization. We also notice that the required constitutive parameters are anisotropic and depend on the radial coordinate $r$ : this complex response is not avoidable, as it is necessary to emulate curved coordinate systems. Finally, we notice that the value of the radial component of the constitutive parameters at the boundary $R_{1}$ of the cloaked region must be zero. This can be understood by considering the ray picture of Fig. 3, which shows how a light ray that goes close to the origin (blue point) in the untransformed coordinate system [Fig. 3(a)] has to travel a greater distance in the transformed coordinate system and yet experience no phase delay [70]. Such a behavior requires that this ray travels faster than the speed of light, and in the limit of the critical ray that crosses the origin, the required phase velocity needs to go to infinity, consistent with the zero value of the constitutive parameters in Eq. (1). In any passive, causal, linear, and time-independent material, according to Kramers-Kronig relations, this can happen only at isolated frequencies, implying a strong frequency dispersion [71].

Given the complex material requirements, such a spherical cloak may become challenging to fabricate and possesses inherent drawbacks. First, the anisotropy and functional dependence of the constitutive parameters is hard to obtain in practice, even with metamaterials. Second, the magnetic response of the cloak should be equal to its electric response, however, a strong magnetic response is difficult to achieve, especially in the optical frequency range, hindering the applicability of the concept to high frequencies. Finally, the superluminal propagation (the requirement of having a phase velocity faster than the speed of light) required in the cloak implies that the concept can ideally work only over a bandwidth of zero measure, i.e., at only one frequency.
Nevertheless, the possibility to bend the flow of energy around an object and conceal it from electromagnetic radiation was demonstrated experimentally at microwave frequencies by Schurig et al. [72], in a frequency range in which strong magnetic responses can be achieved. The design was simplified by considering only one polarization and using the eikonal approximation, which relaxes the constraints on the radial dependence of the cloak parameters at the cost of introducing some mismatch and reflections from the cloak. Such a design makes the cloaking imperfect, however, it maintains the ray-path profile, which is sufficient for the purpose of proving the concept and bending the impinging power around the object. Figure 4 shows a picture of the electromagnetic cloak tested in this experiment, whose design is based on split-ring resonators with tailored radial dependency.

The spherical transformation cloak is an elegant way of achieving invisibility, however, its applicability is limited by two principal drawbacks: (i) the necessity of superluminal light propagation in the cloak, which drastically limits the cloaking bandwidth, and (ii) the complexity of the required constitutive parameters, in particular, the strong required anisotropy and equal electric and magnetic response. These issues can be solved in some specific situations by employing different cloaking techniques: nonEuclidian transformation electrodynamics, which solves problem (i) by sacrificing the phase of the wave; carpet cloaking and its variants, which solve problem (ii) but do not work for free-standing objects or for all angles; and plasmonic cloaking, which solves problem (ii), but is restricted to objects with sizes comparable to the wavelength. We now highlight these other cloaking schemes and their main characteristics.

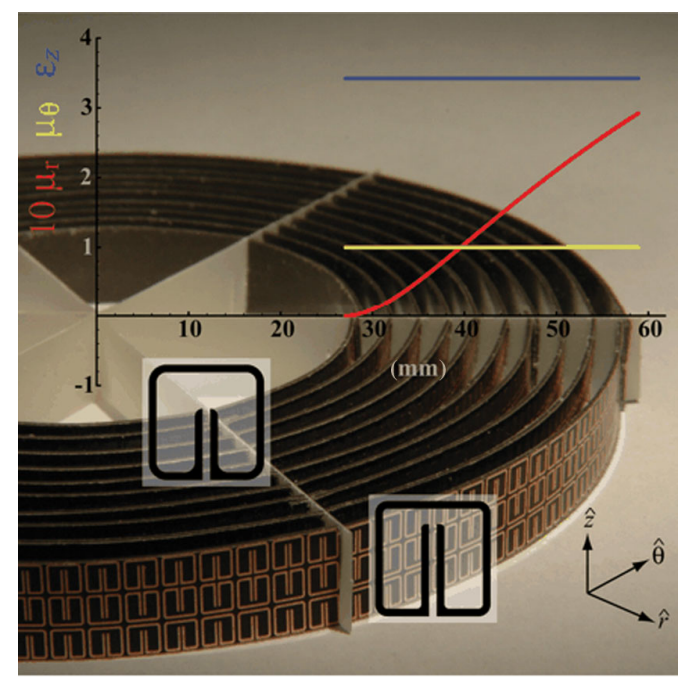

FIG. 4. The electromagnetic cloak based on transformation electrodynamics at microwave frequencies. (C2006 AAAS. Reprinted with permission, Ref. [72]. 


\section{B. Non-Euclidian transformation cloaking}

Non-Euclidian transformation electrodynamics is a technique introduced by Leonhardt and Tyc [73], who proposed using non-Euclidian transformations (for instance, mapping a region of the physical space into the surface of a sphere) in order to avoid singularities in the required cloak-constitutive parameters. The method can be used to completely get rid of the requirement of superluminal propagation. Because of this, cloaking can in principle be made broadband. The principal sacrifice made here to gain bandwidth is that the cloak does not preserve the phase of the impinging wave, unlike the ideal spherical cloak described in the previous section, i.e., the object may become detectable using time-offlight measurement or interferometric techniques. Note, however, that since the human eye is not sensitive to the phase, this compromise may not be a practical problem, even though interference effects can still make the object detectable, as discussed in Ref. [74]. No experimental validation of this concept has been reported to date, probably because the high complexity of practical designs due to the required anisotropic and constitutive parameters for the cloak are still prohibitive; however, this is only a technological challenge, and the method is a promising solution for wideband allangle invisibility.

\section{Carpet cloaking}

Another interesting approach, that greatly simplifies the required parameters for the cloak, has been proposed by $\mathrm{Li}$ and Pendry [75], which introduced a method based on twodimensional quasiconformal transformation electrodynamics. They showed that certain types of transformations may be achieved with dielectric-only materials, reasonable values of permittivity, and weak forms of anisotropy. An example of such a transformation is shown in Fig. 5, in which this method is used to make a mirror with a bump appear perfectly flat, by covering the bump with a carpet cloak, also known as ground-plane cloak. The blue region of the figure represents the volume over which the transformation is applied, while the gray region represents perfect electric conductors, i.e., ideal mirrors. Inside the bump, any object may be hidden. The cloaking method relies on finding the transformation that makes the mirror appear flat, as exemplified in the figure, yielding the

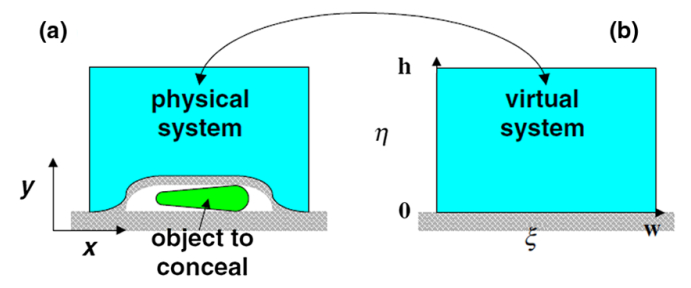

FIG. 5. Carpet cloaking uses a coordinate transformation to make a mirror with a bump appear flat. (C2008 APS. Reprinted with permission, Ref. [75].
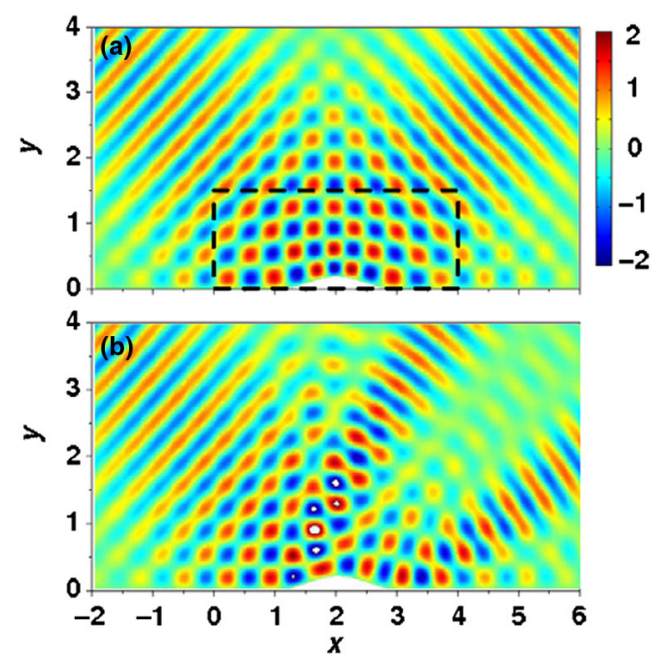

FIG. 6. Numerical simulation of carpet cloaking. (a) Reflection by a PEC mirror with a bump covered by the carpet cloak (contained within the dashed rectangle) and (b) case of the uncovered object. (C)2008 APS. Reprinted with permission, Ref. [75].

required inhomogeneous index distribution for the ground-plane cloak. Figure 6 shows the result of full wave simulations for the scattering of a Gaussian beam by a mirror with a triangular bump, with and without cloak. The beam is reflected as if the mirror where flat in the presence of the cloak, making the bump essentially invisible. Numerous experiments have demonstrated the concept from microwave frequencies to optical frequencies, obtaining cloaking over a wide range of frequencies [76-82]. Figure 7 represents the result of the experiment in Ref. [82] in the cases of blue and red light.

This method also suffers from a certain number of drawbacks. First, it is inherently two dimensional, which makes the concealed object automatically detectable by scattering experiment in the third dimension, as was studied in Ref. [83]. Second, despite the fact that the anisotropy in the cloak is weak, it cannot be approximated to be zero. Isotropic carpet cloaks indeed suffer from the lateral-shift effect [84], making them equivalent to using a second ground plane on top of the bump, which is obviously detectable because there is a shift of the reflected ray for a

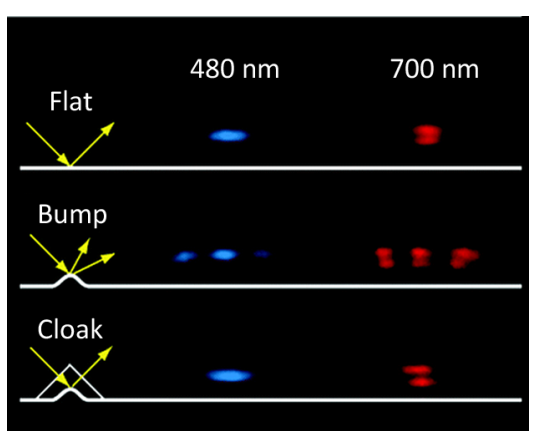

FIG. 7. Experimental validation of carpet cloaking in the visible. (C2011 ACS. Reprinted with permission, Ref. [82]. 
finite width, as if the mirror were closer than it should be. This defeats the initial purpose of trying to get rid of anisotropy: it is simply not possible to have an isotropic carpet cloak, and the weak remaining anisotropy is necessary for invisibility. This remark conducted researchers to ask the following question: instead of aiming at zero anisotropy, may it be possible to aim instead at a specific anisotropic material that we already have in nature, and build alldielectric, broadband carpet cloaks based on natural birefringent materials, such as calcite? After all, we have the degree of freedom of selecting the transformation of choice. Following that idea, it was found that affine transformations can lead to carpet cloaks made of naturally anisotropic materials [85-88], calcite in the visible [87,89], and sapphire at $\mathrm{THz}$ [88]. This method enables relatively broadband carpet cloaking of macroscopic objects with a weak angular sensitivity and no lateral shift, for a given polarization of light. By putting back to back two carpet cloaks, it is also possible to get rid of the ground plane and hide objects in free space either unidirectionally or in multiple directions [90-92]. These carpet-cloak variants work well for large free-standing objects, but only for specific directions and often sacrificing phase preservation.

An interesting direction in the area of carpet cloaking is represented by graded inhomogeneous metasurfaces, which may be able to replace volumetric transformation cloaks to conceal a bump on a ground plane. Recently, using the equivalence principle, interesting ultrathin cloaking metasurfaces have been designed to replace ground-plane cloaks $[93,94]$.

\section{Plasmonic cloaking}

Carpet cloaking and its variants simplify the cloak design by changing the environment of the object to be cloaked (i.e., by exploiting the presence of a ground plane) or by sacrificing all-angle 3D cloaking to work for specific directions of the incident light. Can we achieve all-angle scattering cancellation in free space with simple, homogenous, and isotropic cloaks? The answer is yes, if we focus on suppressing the dominant scattering harmonics radiated from an object, and this is at the basis of plasmonic cloaking. To address the problem formally, let us consider the scattering of a time-harmonic plane wave by an arbitrary object placed at the center of a spherical coordinate system $(r, \theta, \varphi)$. The background medium has permittivity $\varepsilon_{0}$ and permeability $\mu_{0}$. Such a plane wave can be expanded in spherical harmonics under an $e^{j \omega t}$ time convention as [95]

$$
\begin{aligned}
\vec{E}_{i} & =\sum_{n=1}^{\infty} \sum_{m=-n}^{n} a_{n m} \vec{\nabla} \times \vec{\nabla} \times\left(\vec{r} \psi_{n}^{m}\right)-j \omega \mu_{0} b_{n m} \vec{\nabla} \times\left(\vec{r} \psi_{n}^{m}\right), \\
\vec{H}_{i} & =\sum_{n=1}^{\infty} \sum_{m=-n}^{n} b_{n m} \vec{\nabla} \times \vec{\nabla} \times\left(\vec{r} \psi_{n}^{m}\right)-j \omega \varepsilon_{0} a_{n m} \vec{\nabla} \times\left(\vec{r} \psi_{n}^{m}\right),
\end{aligned}
$$

where the $\psi_{n}^{m}$ functions are scalar spherical harmonics, and the coefficients $a_{n m}$ and $b_{n m}$, respectively, describe the amplitude of the incident transverse magnetic (TM) and transverse electric (TE) multipoles of order $(n, m)$. By linearity of Maxwell's equations and orthogonality of each spherical harmonic, the scattered field is composed of all the scattering responses from all TE and TM multipoles [96]:

$$
\begin{aligned}
\vec{E}_{s}= & \sum_{n=1}^{\infty} \sum_{m=-n}^{n} c_{n m}^{\mathrm{TM}} a_{n m} \vec{\nabla} \times \vec{\nabla} \times\left(\vec{r} \psi_{n}^{m}\right) \\
& -j \omega \mu_{0} c_{n m}^{\mathrm{TE}} b_{n m} \vec{\nabla} \times\left(\vec{r} \psi_{n}^{m}\right), \\
\vec{H}_{s}= & \sum_{n=1}^{\infty} \sum_{m=-n}^{n} c_{n m}^{\mathrm{TE}} b_{n m} \vec{\nabla} \times \vec{\nabla} \times\left(\vec{r} \psi_{n}^{m}\right) \\
& -j \omega \varepsilon_{0} c_{n m}^{\mathrm{TM}} a_{n m} \vec{\nabla} \times\left(\vec{r} \psi_{n}^{m}\right) .
\end{aligned}
$$

In Eq. (3), the scattering coefficients $c_{n}^{\mathrm{TE} \text { or TM }}$ are independent of the excitation and depend only on the specific object and on the frequency of operation, quantifying how much scattering is generated in each TE or TM harmonic. Altogether, these coefficients represent the overall visibility of the object, which can rigorously be measured as its total scattering cross section (SCS), given as [96]

$$
\sigma_{s}=\frac{2 \pi}{\left|k_{0}\right|^{2}} \sum_{n=1}^{+\infty} \sum_{m=-n}^{n}(2 n+1)\left(\left|c_{n m}^{\mathrm{TE}}\right|^{2}+\left|c_{n m}^{\mathrm{TM}}\right|^{2}\right),
$$

where $k_{0}$ is the wave number in the background medium. In many situations, the sum in Eq. (4) is dominated by only a few terms. For instance, for an object of characteristic size $a$, only terms up to order $n \approx k_{0} a$ contribute significantly to scattering [97,98]. Plasmonic cloaking is a scattering cancellation technique that uses concentric homogenous and isotropic spherical shells to cancel each of the dominant terms of the scattering cross-section expansion (4) [99-118]. To better understand how it works, let us consider the didactic case of an electrically small dielectric sphere of radius $a$, which is illuminated by a plane wave. In this situation, all the terms with $m \neq 1$ must vanish in Eqs. (3) and (4) [97], and because the sphere is assumed to be electrically small, the only significant term in the sums is the $n=1$ term. We then assume that we cover the dielectric sphere with a spherical shell layer of a different material with permittivity $\varepsilon_{c}$ and outer radius $a_{c}$, obtaining an electrically small core-shell structure whose scattering is effectively described by the dipolar scattering parameters $c_{11}^{\mathrm{TE}}$ and $c_{11}^{\mathrm{TM}}$. They can be calculated using Mie theory [99], and we obtain in a first-order expansion on $k_{0} a$

$$
c_{11}^{\mathrm{TE}}=0, c_{11}^{\mathrm{TM}}=-j\left(k_{0} a_{c}\right)^{3} f\left(\varepsilon_{c}, a / a_{c}\right),
$$

where $f$ is a real-valued function of $\varepsilon_{c}$ and $a / a_{c}$. The TE scattering coefficient is negligible due to the nonmagnetic 
nature of the particle and its subwavelength size, and all the scattering is conveyed by the first TM scattering channel, which is electrically dipolar by nature, and proportional in magnitude to the function $f$. Interestingly, such a function can vanish under the condition [99]

$$
\left(\frac{a}{a_{c}}\right)^{3}=\frac{\left(\varepsilon_{c}-\varepsilon_{0}\right)\left(2 \varepsilon_{c}+\varepsilon\right)}{\left(\varepsilon_{c}-\varepsilon\right)\left(2 \varepsilon_{c}+\varepsilon_{0}\right)}
$$

Equation (6) represents the invisibility condition for a core-shell nanoparticle in the long wavelength limit. The left-hand side of Eq. (6) is bounded between 0 and 1, and therefore the invisibility condition cannot be satisfied for any value of $\varepsilon$ and $\varepsilon_{c}$. The acceptable regions for the core and shell dielectric constants are shown in Fig. 8, where the corresponding value of $a / a_{c}$ is also shown. Notice that any value of permittivity is acceptable for cloaking, however, it is interesting to note that the polarizability of cloak and shell should have opposite values to achieve cloaking. For a dielectric sphere with $\varepsilon>\varepsilon_{0}$, this implies that the cloak requires $\varepsilon \simeq 0$, which may be realized with plasmonic materials, whose permittivity dispersion resembles the one of a plasma, and has a small permittivity around the plasma frequency. The result is that the induced dipole moment in the sphere is exactly compensated by the dipole moment induced in the cloak, which has an opposite sign and identical magnitude, as represented in Fig. 9. Different from all other cloaking methods, both object and cloak, by themselves, scatter the incident wave, and it is only by pairing them that we obtain an invisible object with zero net-induced dipole moment and zero dipolar scattering. This condition for dipolar plasmonic cloaking is also readily understood in the static limit considering Born approximation [119].

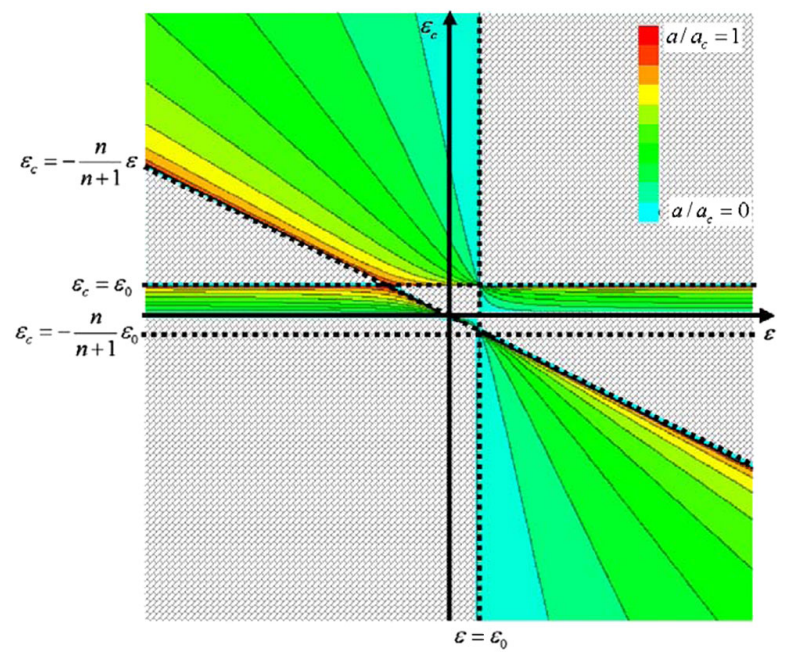

FIG. 8. The cloaking condition for a core-shell nanoparticle in the $\left(\varepsilon, \varepsilon_{c}\right)$ plane. The color contour represents the ratio $a / a_{c} \in[0,1]$. (C2005 APS. Reprinted with permission, Ref. [99].

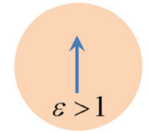

$\vec{P} \neq \overrightarrow{0}$

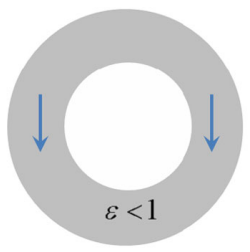

$\vec{P} \neq \overrightarrow{0}$

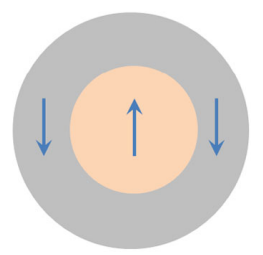

$\vec{P}=\overrightarrow{0}$
FIG. 9. Plasmonic cloaking relies on compensating the polarization of the object using a shell with opposite polarizability. (C2005 APS. Adapted with permission, Ref. [99].

Plasmonic shells can be obtained at optical frequencies by considering noble metals, and at microwave frequencies they can be implemented using metamaterials [111,112]. Bigger objects can be cloaked using several isotropic layers $[105,106]$, and the method is robust to geometric imperfections [107] and generally leads to a larger bandwidth than the transformation-optics approach [120]. Figure 10 shows the experimental validation of plasmonic cloaking at microwave frequencies $(3.1 \mathrm{GHz})$ [114], for a dielectric cylinder covered with a metamaterial cloak. The field distributions measured with and without the cloak show that cloaking is very efficient even in the very near field of the cylinder, see Fig. 11.

\section{E. Mantle cloaking}

Like plasmonic cloaking, mantle cloaking strongly reduces the scattering of an object by canceling the dominant terms in the multipole expansion of the scattered field. The main difference with plasmonic cloaking is that, instead of employing a spherical shell in which an opposite dipole moment is induced, mantle cloaking uses an ultrathin frequency-selective surface (FSS) which is designed so that the induced currents along the surface cancel the scattering from the object to cloak [121-124]. Such a strategy is particularly relevant at microwave frequencies at which frequency-selective surfaces are readily available

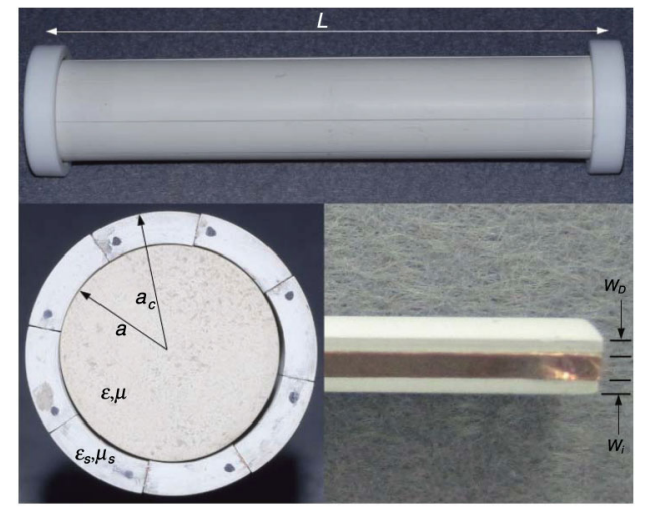

FIG. 10. An experiment on plasmonic cloaking for a dielectric cylinder at microwave frequencies. (C)2012 IOP Publishing. Reprinted with permission, Ref. [114]. 


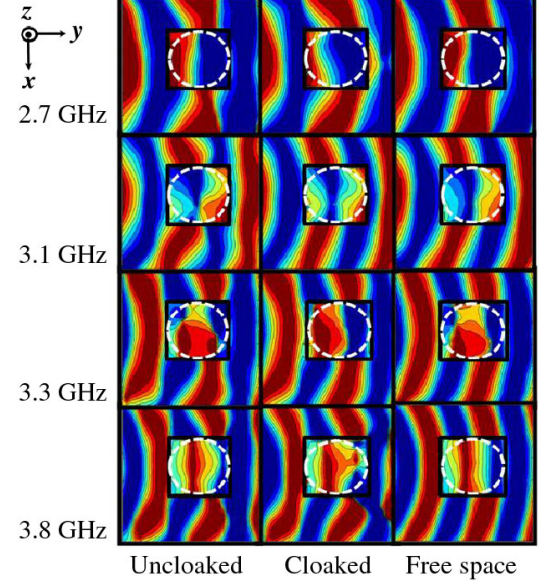

FIG. 11. Near-field measurements (snapshot in time) for the dielectric cylinder of Fig. 10 without the cloak (left), with the cloak (center), and in the case of free space (right). C2012 IOP Publishing. Reprinted with permission, Ref. [114].

and easy to fabricate $[125,126]$. The advantages are the low weight and the thinness of the cloak, which can be conformal to the object. Examples of mantle cloak designs that may be used to control the scattering of a sphere are shown in Fig. 12 [121].

The effect of the surface is to introduce a discontinuity of the tangential magnetic field across its interface. This discontinuity is controlled by the value of the surface impedance $Z_{s}$ of the FSS, yielding the boundary condition

$$
\left.\mathbf{E}_{\mathrm{tan}}\right|_{r=a^{ \pm}}=Z_{s}^{\mathrm{TM}} \mathbf{r} \times\left(\left.\mathbf{H}_{\mathrm{tan}}\right|_{r=a^{+}}-\left.\mathbf{H}_{\mathrm{tan}}\right|_{r=a^{-}}\right) .
$$

Typical designs use periodic metallic patterns deposited on a thin dielectric layer, such as arrays of stripes, patches, holes, or crosses [127]. At THz frequencies, graphene has also been proposed to realize a one-atom-thick mantle cloak [128]. In the example of a small dielectric sphere of permittivity $\varepsilon$ and radius $a$, the required surface impedance for scattering cancellation can be calculated by Mie theory, taking into account the boundary condition (7), and we find [121]

$$
Z_{s}=j \frac{2\left[2+\varepsilon-\gamma^{3}(\varepsilon-1)\right]}{3 \gamma^{3} \omega a \varepsilon_{0}(\varepsilon-1)},
$$
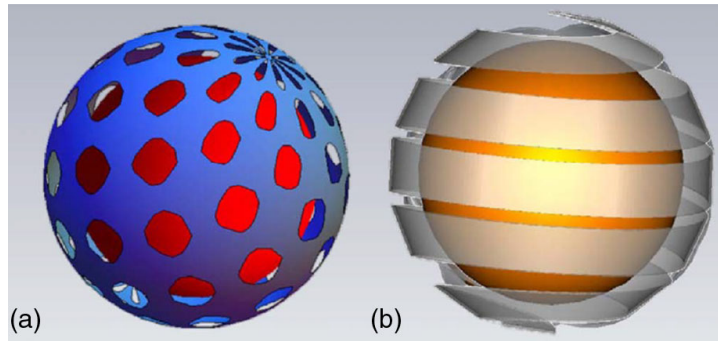

FIG. 12. Possible examples of mantle cloaks for a dielectric sphere. (C2009 APS. Reprinted with permission, Ref. [121].

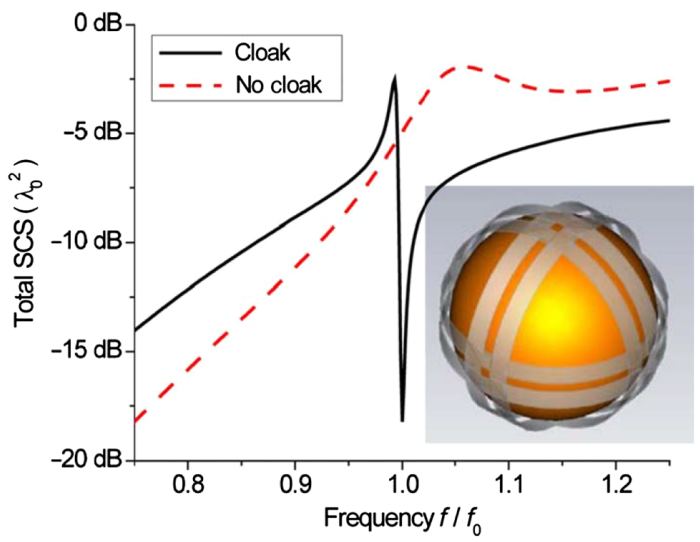

FIG. 13. Significant reduction of the scattering cross section of a dielectric object can be obtained at the design frequency $f_{0}$ using frequency-selective surfaces. (C2009 APS. Reprinted with permission, Ref. [121].

where $\gamma=a / a_{c}$ is the ratio between the sphere and the cloak radii.

Figure 13 shows the example of a dielectric sphere with $\varepsilon=10$, covered by a quasi-isotropic FSS made from six orthogonal interconnected conductive stripes, with $\gamma=0.91$. The plot shows the scattering cross section of the sphere in the cloaked and uncloaked cases versus the frequency in units of the design frequency $f_{0}$, defined as the frequency at which the FSS satisfies condition (8). This realistic design may provide up to $15 \mathrm{~dB}$ scattering reduction at this frequency, for an object diameter of $\lambda / 3$. Mantle cloaking was experimentally validated, obtaining cloaking for a dielectric rod in free space and from all angles [129], and later also verified for conducting objects [130]. Figure 14 shows near-field measurements, and compares the cases of the cloaked cylinder to the uncloaked case and to the case of free space for the dielectric case. Multiple surfaces may be stacked to improve the scattering suppression, broaden the bandwidth, and cloak larger

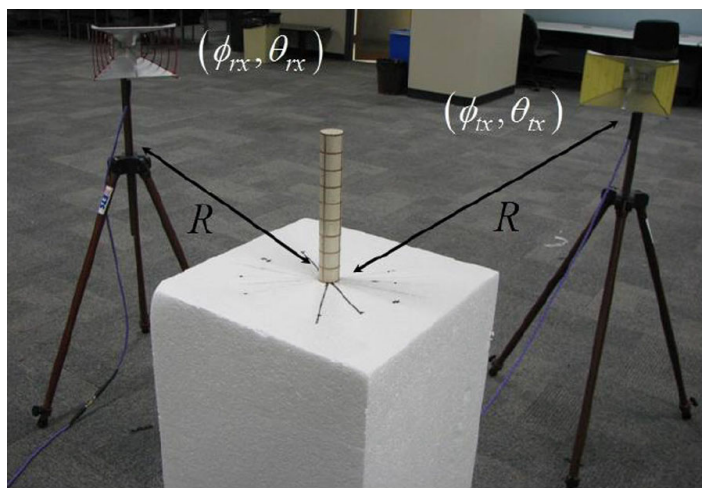

FIG. 14. Experiment on mantle cloaking demonstrating all angle-scattering cancellation for a cylindrical scatterer at microwave frequencies. C2013 IOP Publishing. Reprinted with permission, Ref. [129]. 
TABLE I. Summary of the main advantages and disadvantages of each cloaking method.

\begin{tabular}{lll}
\hline \hline Method & \multicolumn{1}{c}{ Main advantages } & \multicolumn{1}{c}{ Main disadvantages } \\
\hline Spherical transformation cloak & Ideal all-angle cloaking & Zero bandwidth \\
& & High complexity \\
Carpet cloaks and variants & Dielectric-only & 2D cloaking only \\
& Largest bandwidth & Requires mirror, or not all angle \\
Plasmonic and mantle cloaks & Isotropic homogeneous materials & Size limited \\
& Conformal & \\
& Reasonable bandwidth \\
\hline \hline
\end{tabular}

objects. In addition, mantle cloaking is particularly well suited to suppress the scattering from transmitting and receiving antennas, given its conformability and ease of realization $[131,132]$.

\section{F. Comparison of cloaking methods}

In addition to the methods presented above, alternative cloaking techniques are available in the literature. Cloaking using transmission-line networks uses a matched mesh of electromagnetic components to guide the electromagnetic energy within the network which, by itself, does not scatter, and proposes to hide tiny objects in the empty spaces within the mesh [133-137]. Parallel-plate cloaking focuses on a given polarization of the incident field and induces cloaking by routing the electromagnetic power around an object using parallel conductive plates with adiabatically decreasing separation [138-140]. Anomalous resonance methods use the resonance occurring at the interface of complementary media to induce invisibility in a finite region outside the cloak [141-146]. All of these methods are based either on principles similar to transformation electrodynamics, i.e., routing the impinging power around the object, or on the principle of dominant scattering cancellation, in which we let the object be polarized but the polarization of the cloak compensates the one of the object. As they are based on similarly limited phenomena, they possess similar advantages and disadvantages when compared to transformation optics and plasmonic cloaking (see Ref. [147] for more details and a detailed review of these alternative schemes).

All of the cloaking strategies discussed in this section are relevant in different practical situations. They all possess some disadvantages that fundamentally depend on the underlying physics of the cloaking method, as well as technical challenges that need to be solved before they can be used to their full potential. We summarize in Table I the advantages, disadvantages, and/or technical challenges associated with the main cloaking methods discussed in this part.

Looking at Table I, we see that the use of a spherical transformation is an elegant solution to the cloaking problem, as it enables ideal all-angle scattering cancellation regardless of the object and its size. We note, however, that the bandwidth is fundamentally limited to a single frequency, and that the cloak can only be built approximately (for instance, due to the microscopic structure of metamaterials, the phase velocity at the inner boundary will never be exactly infinite). This is a big challenge, as such a cloaking method is sensitive to geometry imperfections [66]. Moreover, the required anisotropy and balanced electric and magnetic response of the cloak is complex, and to date nobody has even attempted to construct such a cloak without simplifying substantially the design, introducing significant unwanted scattering. At optical frequencies, such a cloak may not even be realistic, as strong magnetic responses may not be possible. Carpet cloaks and their variants have the advantage of an all-dielectric design that offers control over the cloak anisotropy and large bandwidth. Initial carpet cloak designs are tailored to minimize anisotropy, and subsequent designs (including the variant of unidirectional calcite cloaks) are tailored to be implementable with natural anisotropic materials. The main drawbacks are the fact that the invisibility is 2D only, and the object is visible in the third dimension, and these methods require either the presence of a ground plane, or work only for specific illumination directions. Finally, plasmonic cloaking is relevant for objects whose size does not exceed a few wavelengths, as it offers imperfect but substantial scattering reduction using simple isotropic and homogeneous cloaks, with the advantage of simplicity in the implementation and a larger bandwidth than 3D transformation cloaks. This is relevant in several application fields. For instance, it has been proposed to cloak sensors and receiving antennas, which are typically subwavelength and relatively narrow band, but would benefit from being furtive and noninvasive. The purpose of cloaking sensors and antennas is to significantly reduce the scattering cross section of such absorptive objects and minimize their perturbation on the probed field [148-163] and their observability. We would also like to mention that hybrid methods have been proposed to optimize bandwidth for a given scattering reduction level, taking advantage of both transformation optics and of a dominant scattering cancellation approach similar to multilayer plasmonic cloaking [164]. However, it appears that a fundamental trade-off exists between the bandwidth and the maximal amount of scattering reduction. In the next section, we analyze the 
inherent constraints and limitations that any cloaking strategy faces, stemming from fundamental constraints as linearity, time independence, causality, and passivity.

\section{FUNDAMENTAL LIMITATIONS}

\section{A. Perfect cloaking for passive absorptive objects is impossible}

Except for the case of perfect vacuum, any passive electromagnetic medium of propagation is bounded to sustain a finite level of absorption. This fundamental limitation is actually a consequence of Kramers-Kronig relations, which hold for any passive causal medium, and imply that dispersion and absorption losses are intimately related [96]. As a result, any passive scatterer will have nonzero material losses, and therefore absorb a nonzero portion of the incident power. The passive invisibility cloaks discussed in the previous section obviously cannot avoid this limitation. Intuitively, it is clear that any absorption of the incident power will somehow translate into a shadow at the back of the object (the forward direction), and therefore complete invisibility is impossible for passive objects. More formally, this is demonstrated by considering the optical theorem $[97,165]$, which states that the total extinction cross section (sum of absorption and scattering) of any scatterer is proportional to the scattering amplitude in the forward direction. As soon as an object has a strictly positive absorption cross section, given the fact that the scattering cross section can be only positive or null, the sum of scattering and absorption cross sections can be only strictly positive. According to the optical theorem, the forward scattering amplitude must then be nonzero, i.e., the object must scatter a certain amount of energy and have a shadow.

This problem is actually related to the problem of seeing without being seen [160]. Is it possible to be under an invisibility cloak and still see the outside world? Seeing is basically a sensing operation, which requires absorbing a small, albeit finite, amount of power from the external field. Therefore, if the cloak is passive, it is not possible to be perfectly invisible and see outside. Some amount of residual scattering must remain. The question, then, is how small can this residual scattering be for a given absorption level? Can this residual scattering be kept very low?

In a recent work [151], the fundamental limitations on scattering and absorption from passive absorptive objects were studied. The main result of this study, shown in Fig. 15, derives the physically allowed region for passive scatterers in blue, for each TE or TM spherical harmonic of order $n$. The partial absorption cross section is represented on the horizontal axis, whereas the vertical axis shows the ratio between absorption and scattering. The plot is made for spherically symmetric objects for simplicity $[m=1$ in Eq. (3)]. For a given absorption level, the scattering in each

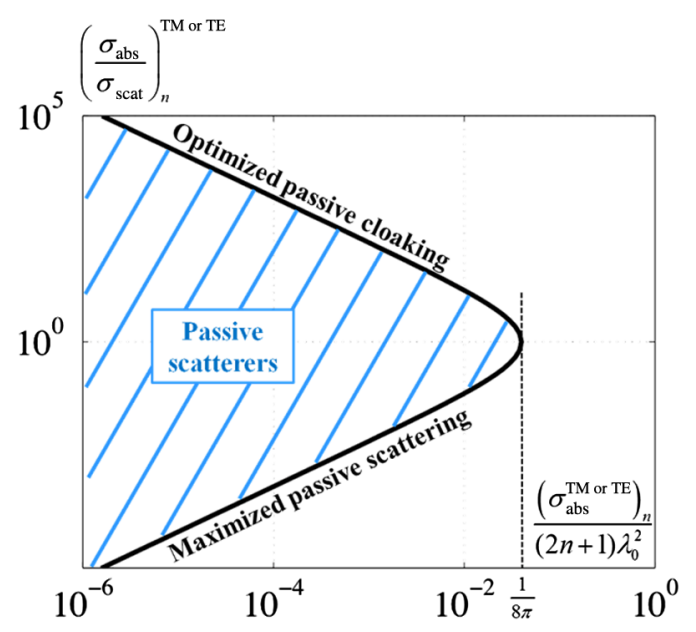

FIG. 15. Passive scatterers are bounded to the blue region, which limits the allowed range for absorption and scattering for each spherical harmonics. (C)2014 APS. Reprinted with permission, Ref. [151].

spherical harmonic is bounded between a minimal and maximal value. When absorption is maximized (rightmost point in the blue region in the figure), the amount of scattering and absorption in a given harmonic are necessarily equal. This illustrates the fact that absorption and scattering are not in general independent of each other. By lowering the absorption level, it is possible to decrease or increase the scattering level within the limit. For sufficiently low absorption levels, we see that scattering can be made extremely low, but can never be identically zero. The black bound in the figure basically quantifies what is possible in terms of scattering reduction for absorptive objects. In summary, consistent with the optical theorem, only objects with identically zero absorption cross section can have identically zero scattering. Absorptive objects have no choice but to scatter within the bound of Fig. 15.

\section{B. Do cloaked objects really scatter less?}

Whenever we introduce a cloak-based on transformation optics, scattering cancellation, or any other technique-we substitute a region of free space with polarizable matter, which scatters and absorbs electromagnetic energy, in a causal way, throughout the electromagnetic spectrum. It is therefore relevant to ask how the introduction of a causal invisibility cloak affects the scattering of an object on a global scale, over broad bandwidths. In fact, although a cloak can be designed to suppress the scattering in a narrow-frequency window, as discussed in the first part of this paper, it is entirely possible that the scattering actually increases in other regions of the frequency spectrum, hence making the cloaked object more visible under broadband illumination, as confirmed by several experimental and numerical observations in the literature. To what extent, therefore, can we say that a cloaked object really scatters less? To address these 
questions in the most general fashion, in Ref. [166] the global response of arbitrary invisibility cloaks over the entire spectrum was studied, deriving fundamental global bounds on cloaking based on general considerations of causality, passivity, and linearity. These results are briefly summarized in this section, and we refer the reader to Refs. [74,166] for additional details.

Different material objects scatter different amounts of energy over the electromagnetic spectrum, depending on their characteristics. Interestingly, however, it was originally demonstrated by Purcell [167], and later generalized in Ref. [168], that if an object is linear and passive, its total scattering cross section $C_{s}$ integrated over the entire spectrum only depends on the static polarizability

$$
\int_{0}^{\infty} C_{s}(\lambda) d \lambda=\pi^{2}\left(\hat{p}_{e}^{*} \cdot \boldsymbol{\alpha}_{\mathbf{e}, s} \cdot \hat{p}_{e}+\hat{p}_{m}^{*} \cdot \boldsymbol{\alpha}_{\mathbf{m}, s} \cdot \hat{p}_{m}\right),
$$

where $\boldsymbol{\alpha}_{\mathbf{e}, s}$ and $\alpha_{\mathbf{m}, s}$ are the electric and magnetic static polarizability tensors, respectively, $\hat{p}_{e}$ denotes the polarization and $\hat{p}_{m}$ the cross-polarization unit vector. Besides, in Eq. (9) and the following discussion, we assume a "lossless" scenario in the sense defined in Ref. [166], namely, material losses are concentrated at isolated frequency points along the spectrum, at which the material parameters diverge (as in the ideal limit of lossless Lorentzian dispersion).

Sum rule (9) indicates that, while frequency dispersion "distributes" the scattering in different regions of the spectrum (for example, an object may scatter more under red light than blue light), the overall scattered energy from static to very high frequencies is uniquely determined by the characteristics of the scatterer at zero frequency. In light of this result, can we predict in general how the static polarizability (and thereby the integrated scattering) changes when a cloak is wrapped around a given object? To answer this question, we can invoke the variational principle derived in Refs. [169,170], which demonstrates the monotonicity of the static electric and magnetic polarizabilities with respect to the local permittivity and permeability. This theorem implies that, whenever we add matter around an object, for example, when we introduce a cloak of generic shape and composition, the static polarizabilities of the overall scatterer are bound to increase if the static permittivity and permeability of the cloak, at all points, are larger than unity relative to the background. Moreover, we recognize that causality and passivity directly imply that the real part of the static relative permittivity $\varepsilon(0)$ cannot be lower than unity. In fact, according to Kramers-Kronig relations, the electric susceptibility at zero frequency becomes [97]

$$
\chi(0)=\operatorname{Re}[\varepsilon(0)]-1=\frac{2}{\pi} \int_{0}^{\infty} \frac{\operatorname{Im}[\varepsilon(\Omega)]}{\Omega} d \Omega,
$$

which is nonnegative if $\operatorname{Im}[\varepsilon] \geq 0$ for every frequency, namely, if the material is passive. Interestingly, similar considerations do not apply to the static magnetic permeability $\mu(0)$, since the function $\mu(\omega)$ loses its physical meaning at moderately high frequencies $[171,172]$, before converging to unity. As a result, different from $\varepsilon(0), \mu(0)$ is allowed to assume values between zero and one [171], which is confirmed by the existence of several natural materials exhibiting static diamagnetism.

The sum rule (9) for the integrated scattering, combined with the monotonicity theorem for the polarizabilities and the nonnegativity of $\chi(0)$, demonstrate that any linear, causal, passive, and nondiamagnetic cloak always increases the static polarizability, and therefore the integrated scattering, of the original uncloaked object. This represents a fundamental global bound on passive cloaking, which indicates that a cloak with the above characteristics, wrapped around a given object, always scatters more, over the entire spectrum, than the original object. In other words, when we design a cloak to reduce the visibility of a scatterer in a narrow-frequency window, we always "pay the price" with increased scattering in other regions of the spectrum. From this perspective, a passive cloak can be interpreted as a device that "redistributes" the scattered energy of an object across the electromagnetic spectrum, such that the integral in Eq. (9) does not decrease. However, it is important to stress that the cloaking bandwidth cannot be widened at will by simply compensating the reduced visibility of an object with higher out-of-band scattering. There are, in fact, additional causality and passivity limitations on the possibility of continuously suppressing the scattering on a given bandwidth, analogous to so-called Bode-Fano limits on the reflection coefficient of passive impedance-matching filters $[173,174]$.

The derived global bound on cloaking confirms and explains why all existing passive cloaking devices tend to strongly increase the scattering outside the narrowfrequency window for which they are designed. In Fig. 16, we show the scattering cross sections, over a wide bandwidth, for typical examples of cloaks belonging to the main categories discussed in this paper, namely, transformation optics, plasmonic, and mantle cloaking. As expected, while all cloaks successfully reduce the visibility of a dielectric sphere at the design frequency, they exhibit much stronger scattering over the rest of the spectrum. Mantle cloaking appears to be the best solution over a global scale, since it requires the smallest amount of additional matter, consistent with the above discussion. In particular, for the considered example, the mantle cloak increases the integrated scattering by 1.75 times, whereas the plasmonic and transformation-optics cloaks increase the integrated scattering by 3.15 and 17.5 times, respectively, as observed in Ref. [166]. These considerations are not only relevant from the scientific standpoint, but are also particularly important to assess the applicability of different cloaking techniques in practical scenarios. In many realworld applications, in fact, ranging from warfare to 


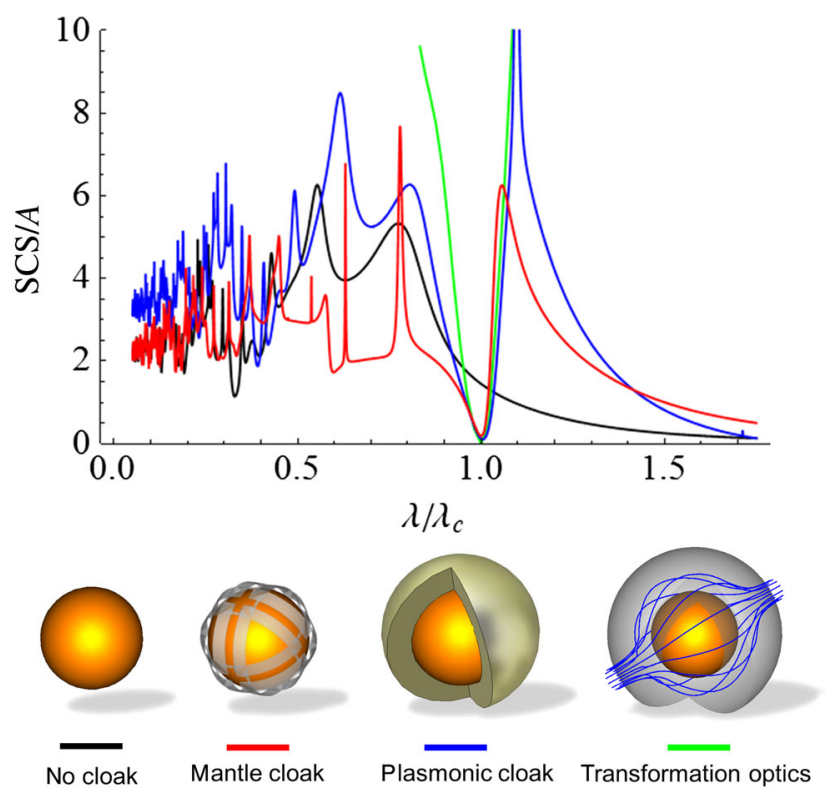

FIG. 16. Normalized scattering cross sections, over a broad range of wavelengths, for the main cloaking devices reviewed in this paper, designed to achieve invisibility at the wavelength $\lambda_{c}$. The normalization factor $A$ is the geometrical cross section of the uncloaked sphere. These results show that existing passive cloaking devices increase the global scattering compared to the uncloaked object. The different cloaks are applied to a spherical dielectric object with relative permittivity $\varepsilon=5$, and diameter $d=\lambda_{c} / 3$. (C)2013 APS. Adapted with permission, Ref. [166].

electromagnetic compatibility and furtive sensing, the broadband properties of cloaking devices are of crucial importance, and therefore a cloak should be designed trying to avoid excessive out-of-band scattering.

The global bound on cloaking discussed in this section may be circumvented if we relax the assumptions over which its derivation is based. First, as mentioned above, if the cloak is diamagnetic at zero frequency, i.e., $0 \leq \mu(0) \leq 1$, the static polarizability of the cloaked object may decrease, which in turn determines a reduction of the integrated scattering. In practice, the required level of diamagnetism to significantly reduce the global scattering can be achieved by including superconducting elements in the cloak, as discussed in Ref. [166] and more extensively in Ref. [74]. Moreover, since magnetization in matter can be interpreted as a form of spatial dispersion of the electric permittivity [171,172], it may be interesting to investigate whether other forms of spatial dispersion may lead to similar cloaking effects as those of static diamagnetism. In fact, it was shown in Refs. [175,176] that a spatially dispersive static permittivity $\varepsilon(0, \mathbf{k})$ can indeed assume finite negative values for momenta $\mathbf{k} \neq \mathbf{0}$ (instead, static permittivity values between zero and one are still strictly forbidden by causality), hence breaking the assumption of non-negative electric susceptibility in Eq. (10).
Another fundamental assumption is the one of causality, which is generally held to be valid in any situation. However, in the scattering problems considered here, causality is defined with respect to the background medium, namely, the response is said to be causal if a signal takes more time reaching a detector in the presence of a scatterer along its path compared to the case when it propagates freely in the background medium. Accordingly, if the background is not a vacuum, but a high-index lossless dielectric, forward scattering may become noncausal and the above global bounds on cloaking do not hold in all situations [both sum rules (9) and (10) are not valid in a noncausal setting]. As a result, it is possible to design broadband cloaks, e.g., a dispersionless air buffer between a high-index background medium and the scatterer as in Ref. [120], which may break the sum rule and achieve much stronger scattering reduction, over wider bandwidths, compared to their counterparts in free space. Interestingly, similar considerations apply to acoustic cloaking [177], which is typically noncausal with respect to the background medium $[178,179]$, and can escape the sum rule.

These considerations can also be related to the locality of the electromagnetic response in typical scattering systems, namely, the fact that the currents induced, at any point, in a material object by an impinging wave depend on the fields at the same point. Any local cloaking scheme aimed at routing a finite signal (i.e., with nonzero bandwidth) around a region of space will inevitably delay the input signal due to causality, leading to finite scattering in the forward direction. In fact, if the response is local, the material at the far side of the cloak will respond to the input signal only when the incident wave front actually reaches that point, i.e., at a time instant that is inevitably delayed compared to the case of free-space propagation without the scatterer (if the background medium is a vacuum). Interestingly, it has been discussed how cloaking for finite signals may be accomplished with a nonlocal response, which may be implemented - at least in principle - with an array of active sources driven by an array of sensors around the scatterer $[180,181]$. In such a design, the cloak response would be strongly nonlocal because the value of every single source is determined by calculations based on field measurements at different points all around the object. Since this approach involves real-time calculations and extra propagation of information, it may be feasible for slowly propagating waves, e.g., in acoustic scattering, whereas it is extremely challenging - if not impossible-for electromagnetic waves, as causality constraints obviously bound also the response of nonlocal materials. Nevertheless, these considerations suggest that the introduction of active elements may open new important directions in the field of cloaking, as we discuss in the following.

The possibility of relaxing the mentioned cloaking bounds by using diamagnetic and superconducting materials or a high-index background, as suggested above, is not 
available, or not practical, in many situations. A different way to circumvent the cloaking limitations is to relax other assumptions made in the above derivation, such as linearity, time independence, or passivity. In particular, consistent with our discussion in the previous paragraph, the use of active cloaking designs holds the promise to outperform conventional passive cloaking techniques, as we discuss in the next section.

\section{OUTLOOK: TOWARDS ACTIVE CLOAKS}

\section{A. Beyond the limitations of passive systems}

The fundamental limitations discussed in the previous section apply to scatterers (i.e., object and cloak) that are made of linear, time-invariant, passive, and causal media. These limitations seem to be rather drastic in terms of bandwidth, sensitivity to absorption losses, and maximal scattering reduction. Because of this, we believe that the future of the science of invisibility and cloaking is to look at ways to circumvent these fundamental limitations and find efficient solutions with broader bandwidth and larger scattering reduction that outperform passive techniques. Among the assumptions of causality, linearity, time invariance, and passivity, the easiest one to break is arguably passivity. Causality - in the sense defined in the previous section-is obviously impossible to break for electromagnetic waves when the background medium is a vacuum. Nonlinearity or time dependence are interesting venues, but such properties typically change the frequency content of the incident signal, causing additional unwanted scattering. Active systems, however, represent the ideal candidates to build efficient broadband invisibility cloaks, as we demonstrate in the rest of this outlook section. They also provide gain, which can be used to our advantage to compensate losses and make perfect invisibility possible, even for absorptive objects, going beyond the limits of Fig. 15.

\section{B. Non-Foster mantle cloaking}

To better understand how active cloaking schemes can break the bandwidth limitations of traditional passive invisibility strategies, we focus on mantle cloaking, and the simple example of an infinite cylinder of radius $a$, relative permittivity $\varepsilon=3$, and relative permeability $\mu=1$, covered by a cylindrical mantle cloak of surface impedance $Z_{s}=j X_{s}$ and radius $a_{c}$. In this cylindrical case, it is possible to show that the transverse magnetic $l=0$ harmonic dominates the overall scattering in the longwavelength limit [122], and the surface reactance $X_{s}$ required to cancel this dominant scattering is found as

$$
X_{s}=\frac{2}{\omega a \gamma(\varepsilon-1)},
$$

where $\gamma=a / a_{c}$. For dielectric cylinders $\varepsilon>1$, this value must be positive $X_{s}>0$, which can be achieved using an inductive FSS (such an inductive surface then compensates for the capacitive nature of the cloaked cylinder). However, the frequency dependence of an inductive surface, typically linear with omega, cannot match the dispersion required by Eq. (11) for broadband cloaking, which is inversely proportional to $\omega$. In fact, there exists no passive FSS that can achieve such a negative dispersion, as it would violate Foster's reactance theorem, according to which the reactance of any passive lumped element must monotonically increase with frequency in a region of low absorption $\partial X_{s}(\omega) / \partial \omega>0$ [182]. What one needs to design to break Foster's theorem is instead an active frequency-selective surface, consistent with Eq. (11), which actually describes an active surface with negative capacitance $Z_{s}=1 / j C \omega$, with $C=(1-\varepsilon) \gamma a / 2$. Such negative non-Foster elements are in fact possible to implement, and have recently attracted significant attention for their potential in broadband electromagnetic wave manipulation using metamaterials at radio frequencies [183].

Designing a metasurface with negative capacitance is quite challenging, due to stability issues inherent to active systems. Using a rigorous approach, our group has proposed a design of an ultrathin metasurface formed by structured metallic patches combined with lumped negative-impedance converters based on complementary metal-oxide-semiconductor technology [184]. A schematic of the design is represented in Fig. 17. Full-wave simulations are performed to predict the scattering from such a structure, fully modeling the coupled electromagnetic and electronic problems.

Figure 18(a) shows the dispersion of the required nonFoster impedance [Eq. (11), red curve] and compares it with the impedance synthesized in our realistic circuit based on negative-impedance converters (green curve) constructed using cross-coupled pairs of bipolar junction transistors (see Ref. [184] for details). The two curves are very close, demonstrating the potential of such circuits to generate the required negative-capacitance dispersion. The blue curve in the figure represents the dispersion of an inductive surface tailored to fulfill condition (11) at the frequency $f_{0}=0.8 \mathrm{GHz}$. We see that, due to the opposite slope, this curve approaches the desired red curve only at its design frequency, illustrating the incapacity of passive designs to generate broadband scattering reduction. On the other hand, the green and red curves are very close to each other in a wide frequency range, allowing for broader scattering cancellation. This is indeed confirmed by looking at the corresponding scattering widths shown in Fig. 18(b), where we also plot the case of the bare cylinder (dashed gray curve). The passive cloak is efficient only at reducing the scattering at its design frequency, with a total reduction of $15 \mathrm{~dB}$ at this frequency. At lower frequencies, however, the passive cloak induces more scattering than the bare object, consistent with the sum rule (9). Using a multilayer cloak, we may be able to induce such a 


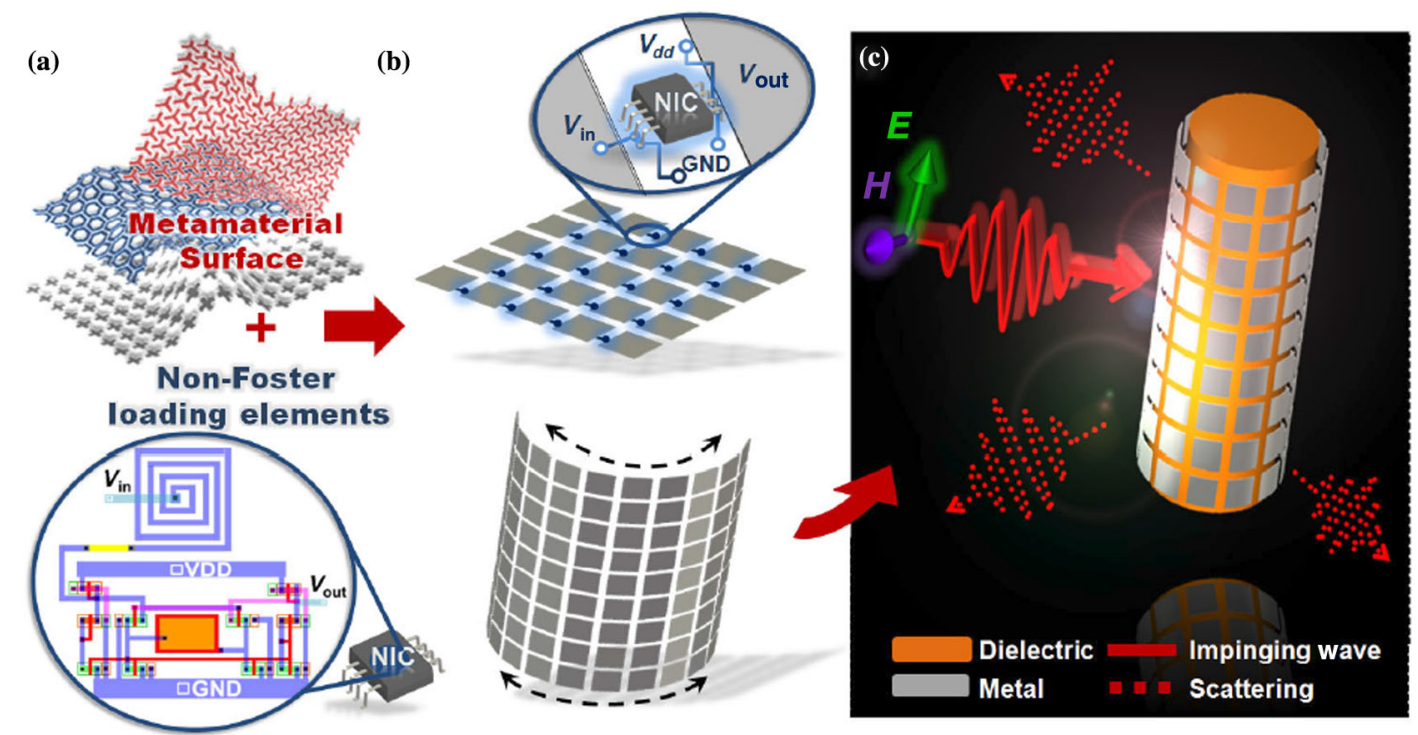

FIG. 17. (a) A metasurface is made of structured metal combined with negative impedance converters powered by a static voltage applied between the drain electrode (VDD) and the ground (GND), assembled (b) to provide a negative capacitive surface, which is ultimately used to implement mantle cloaking (c) over a broad range of frequencies. $d=\lambda_{c} / 3$. C2013 APS. Reprinted with permission, Ref. [184].

scattering dip at multiple frequencies, however, Foster's theorem would force a scattering peak to appear between any two dips [185]. Broadband operation is instead achieved with the active metasurface, which performs much better than the passive cloak in terms of bandwidth, effectively canceling the scattering over almost $900 \mathrm{MHz}$. The setup is fully stable, as confirmed by time-domain simulations of cloaking from finite electromagnetic pulses [184].

\section{Parity-time symmetry and invisibility}

It has been shown in the previous section that active cloaks can break the bandwidth limitations of passive systems. What about the problem of absorption, which imposes a limitation in the absolute value of the scattering reduction level, as shown in Fig. 15? In this section, we explain how active systems, which can provide gain, can also be used to compensate absorption losses, and therefore induce zero absorption cross section. According to the optical theorem, as detailed in Sec. III A, this makes perfect invisibility possible, even for a hidden observer who wants to look at the outside world or a sensor which receives information.

A possible route to realize invisible active systems that can be used as sensors is the use of parity-time (PT) symmetry. PT symmetry is a special type of space-time symmetry that describes the invariance of a system upon the combined action of the Parity operator $P$, which takes the inversions of space, and the time-reversal operator $T$ [42,186-197]. In the one-dimensional case, a PT-symmetric object is invariant upon flipping the object from right to left and (a)

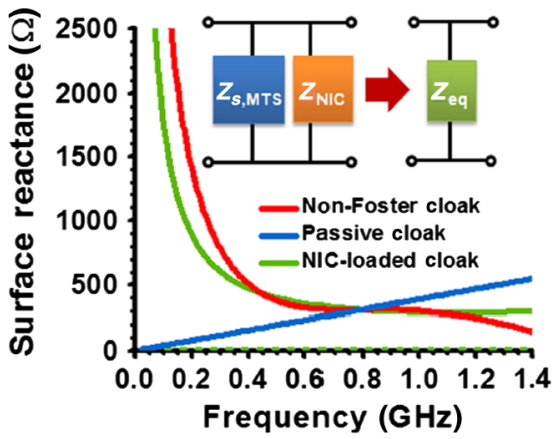

(b)

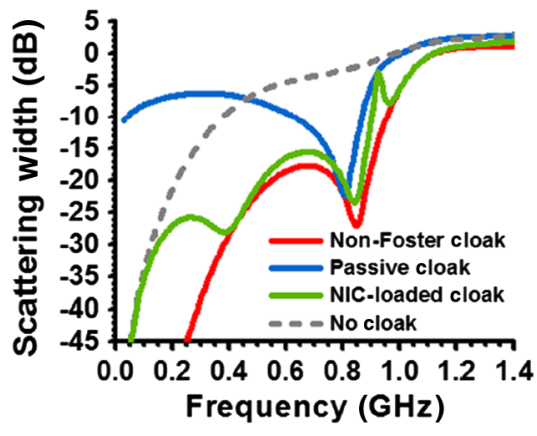

FIG. 18. (a) Surface reactance of different mantle cloaks. The red curve represents the ideal surface reactance for cloaking [see Eq. (11)], while the green curve shows the realistic surface impedance $Z_{\text {eq }}$ designed using commercially available CMOS technology. The non-Foster surface impedance $Z_{\text {eq }}$ is obtained from a passive patch array inductive metasurface (MTS) with surface impedance $Z_{s, \text { MTS}}$, whose gaps are loaded with NIC circuits of electrical impedance $Z_{s, \text { NIC }}$. For comparison, the blue curve represents a passive inductive design. The corresponding scattering widths are shown in (b). (C)2013 APS. Reprinted with permission, Ref. [184]. 


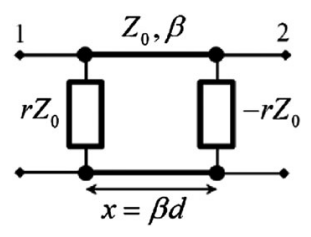

FIG. 19. Example of a PT-symmetric system, obtained by pairing a resistor of value $R=r Z_{0}$ and its time-reversed image, which is a negative resistor of value $-R$. The two circuit elements are connected by a transmission line of impedance $Z_{0}$, propagation constant $\beta$, and length $d$. (C2014 APS. Adapted with permission, Ref. [42].

changing absorption (gain) by an equal amount of gain (loss). For instance, an example of a simple one-dimensional PTsymmetric system is shown in Fig. 19. Two lumped circuit elements are separated by a transmission line segment of characteristic impedance $Z_{0}$ and propagation constant $\beta_{0}$. The circuit element on the left is lossy: it is a simple resistance of value $R=r Z_{0}$, capable of absorbing the power $P=R I^{2}$, where $I$ is the current flowing through it. This circuit can model also simple 1D electromagnetic PT-symmetric geometries. In order to fulfill PT symmetry for the entire system, the element on the right must be the time-reversed image of the element on the left. This is indeed the only way that the $P$ operation, which switches left and right, has the same effect as the $T$ operation, forcing the $P T$ operator to leave the system invariant. The time-reversed image of a resistor of value $R$ is a negative resistor of value $-R$, as it may be understood by applying the time-reversal operation to Ohm's law $U=R I$. Since the voltage $U$ is an even quantity upon time reversal $U \stackrel{T}{\rightarrow} U$, it must be the same for the passive resistor and for its time-reversed image. But since the current $I$ is odd $I \stackrel{T}{\rightarrow}-I$, it is then necessary that $R \stackrel{T}{\rightarrow}-R$. In such a negative resistor, absorption $P=-R I^{2}$ is negative, confirming that the time-reversed image of absorption processes are in fact gain events, sustained by active systems.

This simple example enables us to grasp the relevance of PT symmetry in invisibility problems involving lossy structures. Imagine that the object that we want to hide is the left resistor. Such a resistor can, for instance, model a sensor, which absorbs part of the incident signal to perform a sensing operation. However, because the current induced by the incident signal in the resistor reradiates a scattered field, such a sensing operation would inherently be intrusive and detectable, and this sensor would never be perfectly invisible. As discussed in Sec. III, even by using passive cloaking strategies, we cannot make an absorptive object ideally invisible, and scattering will always occur if the absorption cross section of the cloaked object is nonzero. However, the resistor can be completely cloaked by pairing it with its time-reversed image as in the PT-symmetric configuration of Fig. 19. Such a configuration enables perfect cloaking for the lossy resistor by compensating for its losses and bringing the total absorption cross section to zero. The energy taken out for the sensing operation at the passive resistor is brought back into the system by the active resistor. It is possible to show that such a system can be operated under an invisibility condition, known as an exceptional point in the theory of PT-symmetric systems, that enables zero scattering while maintaining the ability of the passive resistor to absorb power [195]. Such furtive sensors have been implemented experimentally for acoustic waves [197] using properly designed electroacoustic transducers, confirming the theory established in Ref. [42] and the practical relevance of active strategies to implement loss compensation in cloaking, escaping the fundamental limitations of passive systems. Such strategies that exploit PT symmetry may be implemented in more complex scenarios, including cloaking of large three-dimensional objects.

\section{Outlook}

We have shown that active structures provide important degrees of freedom that enable breaking the fundamental limitation rules discussed for passive linear systems in Sec. III, both in terms of bandwidth, scattering reduction level, and sensitivity to absorption losses. While they relax many of the limitations of passive systems, active systems still have to abide by causality, which is reflected in active systems by constraints on their stability. Broadband invisibility-either being based on passive or active schemes-will always be imperfect, and the derivation of fundamental physical bounds for cloaking systems in terms of bandwidth and scattering reduction for active and passive systems is still an open theoretical question. Nevertheless, the above examples show that active systems indeed hold the promise of larger cloaking bandwidths compared to their passive counterparts, and they open the possibility of loss compensation and ideal furtive sensing, which constitute promising research directions in the science of invisibility, bringing us one step closer to the ultimate stealth device.

\section{CONCLUSION}

Many scientific efforts have been made during the past decade to bring the dream of invisibility closer to reality; however, many challenges still stand and need to be overcome to improve the performance of these initial prototypes, particularly in terms of bandwidth and sensitivity to absorption losses. Starting from a concise study of the literature and the basic science of cloaking, we have highlighted the different available methods to induce the invisibility of an arbitrary object, and compared their performance at different levels. We have explained the current limitations that cloaking faces by relating them to basic physical properties of the scattering system, such as linearity and passivity, and derived fundamental limitations that apply to any cloaking scheme, as long as it is based on 
linear, time-independent, passive materials. Active cloaking strategies, which show great potential to alleviate these general physical limitations, were shown to be particularly promising for increasing the cloaking bandwidth and compensating the unwanted scattering due to absorption losses. Altogether, our analysis highlights the necessity to go beyond passive cloaking strategies, which are too constrained in terms of performance, and demonstrates the necessity to focus research efforts on solutions that employ active elements, such as non-Foster metasurfaces or structures with compensated losses, for instance based on PT-symmetry concepts. Other solutions, such as the use of nonlinear or time-dependent materials, may also relax the limitations of conventional cloaking methods.

\section{ACKNOWLEDGMENTS}

This work has been partially supported by the AFOSR Award No. FA9550-13-1-0204, by the NSF CAREER Award No. ECCS-0953311, and the DTRA YIP Award No. HDTRA1-12-1-0022.

R. F. and F. M. have contributed equally to this work.

[1] M. Kerker, Invisible bodies, J. Opt. Soc. Am. 65, 376 (1975).

[2] H. Chew and M. Kerker, Abnormally low electromagnetic scattering cross sections, J. Opt. Soc. Am. 66, 445 (1976).

[3] P. Hertz, Die bewegung eines elektrons unter dem einflusse einer stets gleich gerichteten kraft, Math. Ann. 65, 1 (1907).

[4] D. Bohm and M. Weinstein, The self-oscillations of a charged particle, Phys. Rev. 74, 1789 (1948).

[5] G. H. Goedecke, Classically radiationless motions and possible implications for quantum theory, Phys. Rev. 135, B281 (1964).

[6] B. J. Hoenders, Existence of invisible nonscattering objects and nonradiating sources, J. Opt. Soc. Am. A 14, 262 (1997).

[7] A. D. Boardman, K. Marinov, N. Zheludev, and V. A. Fedotov, Dispersion properties of nonradiating configurations: Finite-difference time-domain modeling, Phys. Rev. E 72, 036603 (2005).

[8] W. K. Kahn and H. Kurss, Minimum-scattering antennas, IEEE Trans. Antennas Propag. 13, 671 (1965).

[9] N. G. Alexopoulos and N. K. Uzunoglu, Electromagnetic scattering from active objects: Invisible scatterers, Appl. Opt. 17, 235 (1978).

[10] P.-S. Kildal, A. A. Kishk, and A. Tengs, Reduction of forward scattering from cylindrical objects using hard surfaces, IEEE Trans. Antennas Propag. 44, 1509 (1996).

[11] A. J. Devaney and G. Sherman, Nonuniqueness in inverse source and scattering problems, IEEE Trans. Antennas Propag. 30, 1034 (1982).

[12] A. J. Devaney, Nonuniqueness in the inverse scattering problem, J. Math. Phys. (N.Y.) 19, 1526 (1978).
[13] A. Greenleaf, M. Lassas, and G. Uhlmann, On nonuniqueness for Calderón's inverse problem, Math. Res. Lett. 10, 685 (2003).

[14] A. Greenleaf, M. Lassas, and G. Uhlmann, Anisotropic conductivities that cannot be detected by EIT, Physiol. Meas. 24, 413 (2003).

[15] C. Caloz and T. Itoh, Electromagnetic Metamaterials: Transmission Line Theory and Microwave Applications (John Wiley \& Sons, Hoboken, 2005).

[16] G. V. Eleftheriades and K. G. Balmain, NegativeRefraction Metamaterials: Fundamental Principles and Applications (John Wiley \& Sons, Hoboken, 2005).

[17] N. Engheta and R. W. Ziolkowski, Electromagnetic Metamaterials: Physics and Engineering Explorations (Wiley-IEEE Press, New York, 2006).

[18] A. K. Sarychev and V. M. Shalaev, Electrodynamics of Metamaterials (World Scientific, Singapore, 2007).

[19] W. Cai and V. M. Shalaev, Optical Metamaterials: Fundamentals and Applications (Springer, New York, 2009).

[20] T. J. Cui, D. R. Smith, and R. Liu, Metamaterials: Theory, Design, and Applications (Springer, New York, 2009).

[21] F. Capolino, Theory and Phenomena of Metamaterials (CRC Press, Boca Raton, 2009).

[22] F. Capolino, Applications of Metamaterials (CRC Press, Boca Raton, 2009).

[23] R. Marqués, F. Martín, and M. Sorolla, Metamaterials with Negative Parameters: Theory, Design and Microwave Applications (John Wiley \& Sons, Hoboken, 2011).

[24] G. Shvets and I. Tsukerman, Plasmonics and Plasmonic Metamaterials: Analysis and Applications (World Scientific, Singapore, 2012).

[25] R. V. Craster and S. Guenneau, Acoustic Metamaterials: Negative Refraction, Imaging, Lensing and Cloaking (Springer, New York, 2012).

[26] V. G. Veselago, The electrodynamics of substances with simultaneously negative values of $\epsilon$ and $\mu$, Sov. Phys. Usp. 10, 509 (1968).

[27] J. B. Pendry, Negative Refraction Makes a Perfect Lens, Phys. Rev. Lett. 85, 3966 (2000).

[28] D. R. Smith, W. J. Padilla, D. C. Vier, S. C. Nemat-Nasser, and S. Schultz, Composite Medium with Simultaneously Negative Permeability and Permittivity, Phys. Rev. Lett. 84, 4184 (2000).

[29] J. Pendry, Optics: Positively negative, Nature (London) 423, 22 (2003).

[30] D. R. Smith, J. B. Pendry, and M.C. K. Wiltshire, Metamaterials and negative refractive index, Science 305, 788 (2004).

[31] M. A. Noginov, H. Li, Y. A. Barnakov, D. Dryden, G. Nataraj, G. Zhu, C. E. Bonner, M. Mayy, Z. Jacob, and E. E. Narimanov, Controlling spontaneous emission with metamaterials, Opt. Lett. 35, 1863 (2010).

[32] Z. Jacob, J.-Y. Kim, G. V. Naik, A. Boltasseva, E. E. Narimanov, and V.M. Shalaev, Engineering photonic density of states using metamaterials, Appl. Phys. B 100, 215 (2010).

[33] A. Alù and N. Engheta, Boosting Molecular Fluorescence with a Plasmonic Nanolauncher, Phys. Rev. Lett. 103, 043902 (2009). 
[34] R. Fleury and A. Alù, Enhanced superradiance in epsilonnear-zero plasmonic channels, Phys. Rev. B 87, 201101 (2013).

[35] M. Silveirinha and N. Engheta, Tunneling of Electromagnetic Energy through Subwavelength Channels and Bends Using $\varepsilon$-Near-Zero Materials, Phys. Rev. Lett. 97, 157403 (2006).

[36] B. Edwards, A. Alù, M. E. Young, M. Silveirinha, and N. Engheta, Experimental Verification of Epsilon-Near-Zero Metamaterial Coupling and Energy Squeezing Using a Microwave Waveguide, Phys. Rev. Lett. 100, 033903 (2008).

[37] R. Fleury and A. Alù, Extraordinary Sound Transmission through Density-Near-Zero Ultranarrow Channels, Phys. Rev. Lett. 111, 055501 (2013).

[38] R. Fleury and A. Alù, Manipulation of electron flow using near-zero index semiconductor metamaterials, Phys. Rev. B 90, 035138 (2014).

[39] A. Alù and N. Engheta, Pairing an epsilon-negative slab with a mu-negative slab: Resonance, tunneling and transparency, IEEE Trans. Antennas Propag. 51, 2558 (2003).

[40] A. Alù, G. D'Aguanno, N. Mattiucci, and M. J. Bloemer, Plasmonic Brewster Angle: Broadband Extraordinary Transmission through Optical Gratings, Phys. Rev. Lett. 106, 123902 (2011).

[41] R. Fleury, D. L. Sounas, C. F. Sieck, M. R. Haberman, and A. Alù, Sound isolation and giant linear nonreciprocity in a compact acoustic circulator, Science 343, 516 (2014).

[42] R. Fleury, D. L. Sounas, and A. Alù, Negative Refraction and Planar Focusing Based on Parity-Time Symmetric Metasurfaces, Phys. Rev. Lett. 113, 023903 (2014).

[43] A. J. Ward and J. B. Pendry, Refraction and geometry in Maxwell's equations, J. Mod. Opt. 43, 773 (1996).

[44] F. L. Teixeira and W. C. Chew, Lattice electromagnetic theory from a topological viewpoint, J. Math. Phys. (N.Y.) 40, 169 (1999).

[45] F. L. Teixeira and W. C. Chew, Differential forms, metrics, and the reflectionless absorption of electromagnetic waves, J. Electromagn. Waves Appl. 13, 665 (1999).

[46] U. Leonhardt, Notes on waves with negative phase velocity, IEEE J. Sel. Top. Quantum Electron. 9, 102 (2003).

[47] H. Chen, B. Hou, S. Chen, X. Ao, W. Wen, and C. T. Chan, Design and Experimental Realization of a Broadband Transformation Media Field Rotator at Microwave Frequencies, Phys. Rev. Lett. 102, 183903 (2009).

[48] D. Schurig, J. B. Pendry, and D. R. Smith, Transformationdesigned optical elements, Opt. Express 15, 14772 (2007).

[49] Y. Liu, T. Zentgraf, G. Bartal, and X. Zhang, Transformational plasmon optics, Nano Lett. 10, 1991 (2010).

[50] A. Alù, F. Bilotti, and L. Vegni, Generalized transmission line equations for bianisotropic materials, IEEE Trans. Antennas Propag. 51, 3134 (2003).

[51] A. Alù, F. Bilotti, and L. Vegni, Method of lines numerical analysis of conformal antennas, IEEE Trans. Antennas Propag. 52, 1530 (2004).

[52] J. B. Pendry, D. Schurig, and D. R. Smith, Controlling electromagnetic fields, Science 312, 1780 (2006).

[53] U. Leonhardt, Optical conformal mapping, Science 312, 1777 (2006).
[54] V. M. Shalaev, Transforming light, Science 322, 384 (2008).

[55] A. Greenleaf, Y. Kurylev, M. Lassas, and G. Uhlmann, Cloaking devices, electromagnetic wormholes, and transformation optics, SIAM Rev. 51, 3 (2009).

[56] H. Chen, C. T. Chan, and P. Sheng, Transformation optics and metamaterials, Nat. Mater. 9, 387 (2010).

[57] B. Zhang, Electrodynamics of transformation-based invisibility cloaking, Light Sci. Appl. 1, e32 (2012).

[58] U. Leonhardt, To invisibility and beyond, Nature (London) 471, 292 (2011).

[59] B. Wood, Metamaterials and invisibility, C.R. Phys. 10, 379 (2009).

[60] P. Sheng, Waves on the horizon, Science 313, 1399 (2006).

[61] U. Leonhardt, Notes on conformal invisibility devices, New J. Phys. 8, 118 (2006).

[62] D. Schurig, J. B. Pendry, and D. R. Smith, Calculation of material properties and ray tracing in transformation media, Opt. Express 14, 9794 (2006).

[63] U. Leonhardt and T. G. Philbin, General relativity in electrical engineering, New J. Phys. 8, 247 (2006).

[64] S. A. Cummer, B.-I. Popa, D. Schurig, D. R. Smith, and J. Pendry, Full-wave simulations of electromagnetic cloaking structures, Phys. Rev. E 74, 036621 (2006).

[65] F. Zolla, S. Guenneau, A. Nicolet, and J. B. Pendry, Electromagnetic analysis of cylindrical invisibility cloaks and the mirage effect, Opt. Lett. 32, 1069 (2007).

[66] Z. Ruan, M. Yan, C. W. Neff, and M. Qiu, Ideal Cylindrical Cloak: Perfect but Sensitive to Tiny Perturbations, Phys. Rev. Lett. 99, 113903 (2007).

[67] H. Chen, B.-I. Wu, B. Zhang, and J. A. Kong, Electromagnetic Wave Interactions with a Metamaterial Cloak, Phys. Rev. Lett. 99, 063903 (2007).

[68] B. Zhang, H. Chen, B.-I. Wu, Y. Luo, L. Ran, and J. A. Kong, Response of a cylindrical invisibility cloak to electromagnetic waves, Phys. Rev. B 76, 121101 (2007).

[69] A. D. Yaghjian and S. Maci, Alternative derivation of electromagnetic cloaks and concentrators, New J. Phys. 10, 115022 (2008).

[70] J. Perczel, T. Tyc, and U. Leonhardt, Invisibility cloaking without superluminal propagation, New J. Phys. 13, 083007 (2011).

[71] J. S. Toll, Causality and the dispersion relation: Logical foundations, Phys. Rev. 104, 1760 (1956).

[72] D. Schurig, J. J. Mock, B. J. Justice, S. A. Cummer, J. B. Pendry, A. F. Starr, and D. R. Smith, Metamaterial electromagnetic cloak at microwave frequencies, Science 314, 977 (2006).

[73] U. Leonhardt and T. Tyc, Broadband invisibility by nonEuclidean cloaking, Science 323, 110 (2009).

[74] F. Monticone and A. Alù, Physical bounds on electromagnetic invisibility and the potential of superconducting cloaks, Photon. Nanostr. Fundam. Appl. 12, 330 (2014).

[75] J. Li and J. B. Pendry, Hiding under the Carpet: A New Strategy for Cloaking, Phys. Rev. Lett. 101, 203901 (2008).

[76] R. Liu, C. Ji, J. J. Mock, J. Y. Chin, T. J. Cui, and D. R. Smith, Broadband ground-plane cloak, Science 323, 366 (2009). 
[77] J. Valentine, J. Li, T. Zentgraf, G. Bartal, and X. Zhang, An optical cloak made of dielectrics, Nat. Mater. 8, 568 (2009).

[78] J. H. Lee, J. Blair, V. A. Tamma, Q. Wu, S. J. Rhee, C. J. Summers, and W. Park, Direct visualization of optical frequency invisibility cloak based on silicon nanorod array, Opt. Express 17, 12922 (2009).

[79] L. H. Gabrielli, J. Cardenas, C. B. Poitras, and M. Lipson, Silicon nanostructure cloak operating at optical frequencies, Nat. Photonics 3, 461 (2009).

[80] T. Ergin, N. Stenger, P. Brenner, J. B. Pendry, and M. Wegener, Three-dimensional invisibility cloak at optical wavelengths, Science 328, 337 (2010).

[81] H. F. Ma and T. J. Cui, Three-dimensional broadband ground-plane cloak made of metamaterials, Nat. Commun. 1, 21 (2010).

[82] M. Gharghi, C. Gladden, T. Zentgraf, Y. Liu, X. Yin, J. Valentine, and X. Zhang, A carpet cloak for visible light, Nano Lett. 11, 2825 (2011).

[83] J. C. Halimeh, T. Ergin, J. Mueller, N. Stenger, and M. Wegener, Photorealistic images of carpet cloaks, Opt. Express 17, 19328 (2009).

[84] B. Zhang, T. Chan, and B.-I. Wu, Lateral Shift Makes a Ground-Plane Cloak Detectable, Phys. Rev. Lett. 104, 233903 (2010).

[85] Y. Luo, J. Zhang, H. Chen, L. Ran, B.-I. Wu, and J. A. Kong, A rigorous analysis of plane-transformed invisibility cloaks, IEEE Trans. Antennas Propag. 57, 3926 (2009).

[86] S. Xi, H. Chen, B.-I. Wu, and J.-A. Kong, One-directional perfect cloak created with homogeneous material, IEEE Microwave Wireless Compon. Lett. 19, 131 (2009).

[87] X. Chen, Y. Luo, J. Zhang, K. Jiang, J. B. Pendry, and S. Zhang, Macroscopic invisibility cloaking of visible light, Nat. Commun. 2, 176 (2011).

[88] D. Liang, J. Gu, J. Han, Y. Yang, S. Zhang, and W. Zhang, Robust large dimension terahertz cloaking, Adv. Mater. 24, 916 (2012).

[89] B. Zhang, Y. Luo, X. Liu, and G. Barbastathis, Macroscopic Invisibility Cloak for Visible Light, Phys. Rev. Lett. 106, 033901 (2011).

[90] H. Chen and B. Zheng, Broadband polygonal invisibility cloak for visible light, Sci. Rep. 2, 255 (2012).

[91] N. Landy and D. R. Smith, A full-parameter unidirectional metamaterial cloak for microwaves, Nat. Mater. 12, 25 (2013).

[92] Y. Urzhumov, N. Landy, T. Driscoll, D. Basov, and D. R. Smith, Thin low-loss dielectric coatings for free-space cloaking, Opt. Lett. 38, 1606 (2013).

[93] N. M. Estakhri, C. Argyropoulos, and A. Alù, Graded metascreens to enable a new degree of nanoscale light management, Phil. Trans. R. Soc. A 373, 20140351 (2015).

[94] N. M. Estakhri and A. Alù, Ultra-thin unidirectional carpet cloak and wavefront reconstruction with graded metasurfaces, IEEE Antennas Wireless Propag. Lett. 13, 1775 (2014).

[95] C. H. Papas, Theory of Electromagnetic Wave Propagation (Courier Dover Publications, New York, 2013).

[96] J. D. Jackson, Classical Electrodynamics (Wiley, New York, 1998).

[97] C. F. Bohren and D. R. Huffman, Absorption and Scattering of Light by Small Particles (John Wiley \& Sons, New York, 2008).
[98] A. Alù and N. Engheta, Polarizabilities and effective parameters for collections of spherical nanoparticles formed by pairs of concentric double-negative, singlenegative, and/or double-positive metamaterial layers, J. Appl. Phys. 97, 094310 (2005).

[99] A. Alù and N. Engheta, Achieving transparency with plasmonic and metamaterial coatings, Phys. Rev. E 72, 016623 (2005).

[100] P. Ball, Engineers devise invisibility shield, Nat. News, doi:10.1038/news050228-1 (2005).

[101] A. Alù and N. Engheta, Plasmonic and metamaterial cloaking: Physical mechanisms and potentials, J. Opt. A 10, 093002 (2008).

[102] P.-Y. Chen, J. Soric, and A. Alù, Invisibility and cloaking based on scattering cancellation, Adv. Mater. 24, OP281 (2012).

[103] A. Alù and N. Engheta, Plasmonic materials in transparency and cloaking problems: Mechanism, robustness, and physical insights, Opt. Express 15, 3318 (2007).

[104] A. Alù and N. Engheta, Cloaking and transparency for collections of particles with metamaterial and plasmonic covers, Opt. Express 15, 7578 (2007).

[105] A. Alù and N. Engheta, Multifrequency Optical Invisibility Cloak with Layered Plasmonic Shells, Phys. Rev. Lett. 100, 113901 (2008).

[106] A. Alù and N. Engheta, Theory and potentials of multilayered plasmonic covers for multi-frequency cloaking, New J. Phys. 10, 115036 (2008).

[107] S. Tricarico, F. Bilotti, A. Alù, and L. Vegni, Plasmonic cloaking for irregular objects with anisotropic scattering properties, Phys. Rev. E 81, 026602 (2010).

[108] E. Kallos, C. Argyropoulos, Y. Hao, and A. Alù, Comparison of frequency responses of cloaking devices under nonmonochromatic illumination, Phys. Rev. B 84, 045102 (2011).

[109] A. Alù, D. Rainwater, and A. Kerkhoff, Plasmonic cloaking of cylinders: Finite length, oblique illumination and cross-polarization coupling, New J. Phys. 12, 103028 (2010).

[110] M. G. Silveirinha, A. Alù, and N. Engheta, Cloaking mechanism with antiphase plasmonic satellites, Phys. Rev. B 78, 205109 (2008).

[111] M. G. Silveirinha, A. Alù, and N. Engheta, Parallel-plate metamaterials for cloaking structures, Phys. Rev. E 75, 036603 (2007).

[112] M. G. Silveirinha, A. Alù, and N. Engheta, Infrared and optical invisibility cloak with plasmonic implants based on scattering cancellation, Phys. Rev. B 78, 075107 (2008).

[113] B. Edwards, A. Alù, M. G. Silveirinha, and N. Engheta, Experimental Verification of Plasmonic Cloaking at Microwave Frequencies with Metamaterials, Phys. Rev. Lett. 103, 153901 (2009).

[114] D. Rainwater, A. Kerkhoff, K. Melin, J. C. Soric, G. Moreno, and A. Alù, Experimental verification of threedimensional plasmonic cloaking in free-space, New J. Phys. 14, 013054 (2012).

[115] R. Fleury and A. Alù, Quantum cloaking based on scattering cancellation, Phys. Rev. B 87, 045423 (2013). 
[116] M. D. Guild, M. R. Haberman, and A. Alù, Plasmonic cloaking and scattering cancelation for electromagnetic and acoustic waves, Wave Motion 48, 468 (2011).

[117] M. D. Guild, M. R. Haberman, and A. Alù, Plasmonic-type acoustic cloak made of a bilaminate shell, Phys. Rev. B 86, 104302 (2012).

[118] M. D. Guild, A. Alù, and M. R. Haberman, Cancellation of acoustic scattering from an elastic sphere, J. Acoust. Soc. Am. 129, 1355 (2011).

[119] R. Fleury and A. Alù, Exotic properties and potential applications of quantum metamaterials, Appl. Phys. A 109, 781 (2012).

[120] A. Alù and N. Engheta, Effects of size and frequency dispersion in plasmonic cloaking, Phys. Rev. E 78, 045602 (2008).

[121] A. Alù, Mantle cloak: Invisibility induced by a surface, Phys. Rev. B 80, 245115 (2009).

[122] P.-Y. Chen and A. Alù, Mantle cloaking using thin patterned metasurfaces, Phys. Rev. B 84, 205110 (2011).

[123] P.-Y. Chen, F. Monticone, and A. Alù, Suppressing the electromagnetic scattering with an helical mantle cloak, IEEE Antennas Wireless Propag. Lett. 10, 1598 (2011).

[124] Y. R. Padooru, A. B. Yakovlev, P.-Y. Chen, and A. Alù, Line-source excitation of realistic conformal metasurface cloaks, J. Appl. Phys. 112, 104902 (2012).

[125] B. A. Munk, Frequency Selective Surfaces: Theory and Design (John Wiley \& Sons, New York, 2005).

[126] S. Tretyakov, Analytical Modeling in Applied Electromagnetics (Artech House, Boston, 2003).

[127] Y. R. Padooru, A. B. Yakovlev, P.-Y. Chen, and A. Alù, Analytical modeling of conformal mantle cloaks for cylindrical objects using sub-wavelength printed and slotted arrays, J. Appl. Phys. 112, 034907 (2012).

[128] P.-Y. Chen and A. Alù, Atomically thin surface cloak using graphene monolayers, ACS Nano 5, 5855 (2011).

[129] J. C. Soric, P. Y. Chen, A. Kerkhoff, D. Rainwater, K. Melin, and A. Alù, Demonstration of an ultralow profile cloak for scattering suppression of a finite-length rod in free space, New J. Phys. 15, 033037 (2013).

[130] R. S. Schofield, J. C. Soric, D. Rainwater, A. Kerkhoff, and A. Alù, Scattering suppression and wideband tunability of a flexible mantle cloak for finite-length conducting rods, New J. Phys. 16, 063063 (2014).

[131] J. C. Soric, A. Monti, A. Toscano, F. Bilotti, and A. Alù, Antenna blockage reduction using metasurface cloaks (to be published).

[132] J. C. Soric, A. Monti, A. Toscano, F. Bilotti, and A. Alù, Multiband and wideband multilayer mantle cloaks, IEEE Trans. Antennas Propag. 63, 3235 (2015).

[133] P. Alitalo, O. Luukkonen, L. Jylha, J. Venermo, and S. A. Tretyakov, Transmission-line networks cloaking objects from electromagnetic fields, IEEE Trans. Antennas Propag. 56, 416 (2008).

[134] P. Alitalo, O. Luukkonen, L. Jylha, J. Venermo, and S. A. Tretyakov, Correction to "Transmission-line networks cloaking objects from electromagnetic fields" [Feb 08 416-424], IEEE Trans. Antennas Propag. 56, 918 (2008).

[135] P. Alitalo, S. Ranvier, J. Vehmas, and S. Tretyakov, A microwave transmission-line network guiding electromagnetic fields through a dense array of metallic objects, Metamaterials 2, 206 (2008).

[136] P. Alitalo and S. Tretyakov, Electromagnetic cloaking with metamaterials, Mater. Today 12, 22 (2009).

[137] P. Alitalo, F. Bongard, J.-F. Zürcher, J. Mosig, and S. Tretyakov, Experimental verification of broadband cloaking using a volumetric cloak composed of periodically stacked cylindrical transmission-line networks, Appl. Phys. Lett. 94, 014103 (2009).

[138] S. Tretyakov, P. Alitalo, O. Luukkonen, and C. Simovski, Broadband Electromagnetic Cloaking of Long Cylindrical Objects, Phys. Rev. Lett. 103, 103905 (2009).

[139] P. Alitalo and S. A. Tretyakov, Electromagnetic cloaking of strongly scattering cylindrical objects by a volumetric structure composed of conical metal plates, Phys. Rev. B 82, 245111 (2010).

[140] P. Alitalo, A. E. Culhaoglu, A. V. Osipov, S. Thurner, E. Kemptner, and S. A. Tretyakov, Bistatic scattering characterization of a three-dimensional broadband cloaking structure, J. Appl. Phys. 111, 034901 (2012).

[141] G. W. Milton and N.-A. P. Nicorovici, On the cloaking effects associated with anomalous localized resonance, Proc. R. Soc. A 462, 3027 (2006).

[142] G. W. Milton, N.-A. P. Nicorovici, R. C. McPhedran, and V. A. Podolskiy, A proof of superlensing in the quasistatic regime, and limitations of superlenses in this regime due to anomalous localized resonance, Proc. R. Soc. A 461, 3999 (2005).

[143] N. A. Nicorovici, G. W. Milton, R. C. McPhedran, and L. C. Botten, Quasistatic cloaking of two-dimensional polarizable discrete systems by anomalous resonance, Opt. Express 15, 6314 (2007).

[144] N.-a. P. Nicorovici, R. C. McPhedran, S. Enoch, and G. Tayeb, Finite wavelength cloaking by plasmonic resonance, New J. Phys. 10, 115020 (2008).

[145] Y. Lai, H. Chen, Z.-Q. Zhang, and C. T. Chan, Complementary Media Invisibility Cloak that Cloaks Objects at a Distance Outside the Cloaking Shell, Phys. Rev. Lett. 102, 093901 (2009).

[146] G. Zheng, X. Heng, and C. Yang, A phase conjugate mirror inspired approach for building cloaking structures with left-handed materials, New J. Phys. 11, 033010 (2009).

[147] R. Fleury and A. Alù, Cloaking and invisibility: A review, FERMAT 1, 7 (2014).

[148] A. Alù, Cloaking a Sensor, Phys. Rev. Lett. 102, 233901 (2009).

[149] A. Alù and N. Engheta, Cloaking a receiving antenna or a sensor with plasmonic metamaterials, Metamaterials 4, 153 (2010).

[150] A. Alù and S. Maslovski, Power relations and a consistent analytical model for receiving wire antennas, IEEE Trans. Antennas Propag. 58, 1436 (2010).

[151] R. Fleury, J. Soric, and A. Alù, Physical bounds on absorption and scattering for cloaked sensors, Phys. Rev. B 89, 045122 (2014).

[152] C. A. Valagiannopoulos and N. L. Tsitsas, Integral equation analysis of a low-profile receiving planar microstrip antenna with a cloaking superstrate, Radio Sci. (to be published). 
[153] A. Monti, J. Soric, A. Alu, F. Bilotti, A. Toscano, and L. Vegni, Overcoming mutual blockage between neighboring dipole antennas using a low-profile patterned metasurface, IEEE Antennas Wireless Propag. Lett. 11, 1414 (2012).

[154] J. C. Soric, R. Fleury, A. Monti, A. Toscano, F. Bilotti, and A. Alu, Controlling scattering and absorption with metamaterial covers, IEEE Trans. Antennas Propag. 62, 4220 (2014).

[155] A. Alù and N. Engheta, Cloaked Near-Field Scanning Optical Microscope Tip for Noninvasive Near-Field Imaging, Phys. Rev. Lett. 105, 263906 (2010).

[156] F. Bilotti, S. Tricarico, F. Pierini, and L. Vegni, Cloaking apertureless near-field scanning optical microscopy tips, Opt. Lett. 36, 211 (2011).

[157] F. Bilotti, F. Pierini, and L. Vegni, Employment of metamaterial cloaks to enhance the resolution of nearfield scanning optical microscopy systems based on aperture tips, Metamaterials 5, 119 (2011).

[158] P. Fan, U. K. Chettiar, L. Cao, F. Afshinmanesh, N. Engheta, and M. L. Brongersma, An invisible metalsemiconductor photodetector, Nat. Photonics 6, 380 (2012).

[159] A. Greenleaf, Y. Kurylev, M. Lassas, and G. Uhlmann, Cloaking a sensor via transformation optics, Phys. Rev. E 83, 016603 (2011).

[160] F. J. García de Abajo, Seeing without being seen, Physics 2, 47 (2009).

[161] R. Fleury and A. Alù, Furtive quantum sensing using matter-wave cloaks, Phys. Rev. B 87, 201106 (2013).

[162] A. Greenleaf, Y. Kurylev, M. Lassas, U. Leonhardt, and G. Uhlmann, Cloaked electromagnetic, acoustic, and quantum amplifiers via transformation optics, Proc. Natl. Acad. Sci. U.S.A. 109, 10169 (2012).

[163] T. Xu, X.-F. Zhu, B. Liang, Y. Li, X.-Y. Zou, and J.-C. Cheng, Scattering reduction for an acoustic sensor using a multilayered shell comprising a pair of homogeneous isotropic single-negative media, Appl. Phys. Lett. 101, 033509 (2012).

[164] S. Xu et al., Experimental Demonstration of a Free-Space Cylindrical Cloak without Superluminal Propagation, Phys. Rev. Lett. 109, 223903 (2012).

[165] A. Alù and N. Engheta, How does zero forward-scattering in magnetodielectric nanoparticles comply with the optical theorem? J. Nanophoton. 4, 041590 (2010).

[166] F. Monticone and A. Alù, Do Cloaked Objects Really Scatter Less? Phys. Rev. X 3, 041005 (2013).

[167] E. M. Purcell, On the absorption and emission of light by interstellar grains, Astrophys. J. 158, 433 (1969).

[168] M. Gustafsson, C. Sohl, and G. Kristensson, Physical limitations on antennas of arbitrary shape, Proc. R. Soc. A 463, 2589 (2007).

[169] D. S. Jones, Scattering by inhomogeneous dielectric particles, Q. J. Mech. Appl. Math. 38, 135 (1985).

[170] D. Sjöberg, Variational principles for the static electric, and magnetic polarizabilities of anisotropic media with perfect electric conductor inclusions, J. Phys. A 42, 335403 (2009).

[171] L. Landau and E. Lifshitz, Electrodynamics of Continuous Media (Pergamon Press, Oxford, 1966).
[172] F. Monticone and A. Alù, The Quest for Optical Magnetism: From Split-Ring Resonators to Plasmonic Nanoparticles and Nanoclusters, J. Mater. Chem. C 2, 9059 (2014).

[173] R. M. Fano, Theoretical limitations on the broadband matching of arbitrary impedances, J. Franklin Inst. 249, 57 (1950).

[174] D. M. Pozar, Microwave Engineering (Wiley, New York, 2011).

[175] O. Dolgov, D. Kirzhnits, and E. Maksimov, On an admissible sign of the static dielectric function of matter, Rev. Mod. Phys. 53, 81 (1981).

[176] O. V. Dolgov, D. A. Kirzhnits, and V. V. Losyakov, Admissible values of permittivity and magnetic permeability of matter, J. Exp. Theor. Phys. 56, 1095 (1982).

[177] A. N. Norris, Acoustic cloaking, Acoustics Today 11, 38 (2015).

[178] A. N. Norris, Acoustic cloaking theory, Proc. R. Soc. A 464, 2411 (2008).

[179] A. N. Norris, Acoustic integrated extinction Proc. R. Soc. A 471, 20150008 (2015).

[180] D. A. B. Miller, On perfect cloaking, Opt. Express 14, 12457 (2006).

[181] M. Selvanayagam and G. V. Eleftheriades, Experimental Demonstration of Active Electromagnetic Cloaking, Phys. Rev. X 3, 041011 (2013).

[182] R. M. Foster, A reactance theorem, Bell Syst. Tech. J. 3, 259 (1924).

[183] S. Hrabar, I. Krois, I. Bonic, and A. Kiricenko, Negative capacitor paves the way to ultrabroadband metamaterials, Appl. Phys. Lett. 99, 254103 (2011).

[184] P.-Y. Chen, C. Argyropoulos, and A. Alù, Broadening the Bandwidth of Metamaterial Cloaks with Non-Foster Metasurfaces, Phys. Rev. Lett. 111, 233001 (2013).

[185] F. Monticone, C. Argyropoulos, and A. Alù, Multilayered Plasmonic Covers for Comblike Scattering Response and Optical Tagging, Phys. Rev. Lett. 110, 113901 (2013).

[186] C. M. Bender and S. Boettcher, Real Spectra in NonHermitian Hamiltonians Having PT Symmetry, Phys. Rev. Lett. 80, 5243 (1998).

[187] K. G. Makris, R. El-Ganainy, D. N. Christodoulides, and Z. H. Musslimani, Beam Dynamics in PT Symmetric Optical Lattices, Phys. Rev. Lett. 100, 103904 (2008).

[188] Z. H. Musslimani, K. G. Makris, R. El-Ganainy, and D. N. Christodoulides, Optical Solitons in PT Periodic Potentials, Phys. Rev. Lett. 100, 030402 (2008).

[189] P. Ambichl, K. G. Makris, L. Ge, Y. Chong, A. D. Stone, and S. Rotter, Breaking of PT Symmetry in Bounded and Unbounded Scattering Systems, Phys. Rev. X 3, 041030 (2013).

[190] S. Klaiman, U. Günther, and N. Moiseyev, Visualization of Branch Points in PT-Symmetric Waveguides, Phys. Rev. Lett. 101, 080402 (2008).

[191] S. Longhi, Bloch Oscillations in Complex Crystals with PT Symmetry, Phys. Rev. Lett. 103, 123601 (2009).

[192] Y. D. Chong, L. Ge, and A. D. Stone, PT-Symmetry Breaking and Laser-Absorber Modes in Optical Scattering Systems, Phys. Rev. Lett. 106, 093902 (2011). 
[193] C. E. Rüter, K. G. Makris, R. El-Ganainy, D. N. Christodoulides, M. Segev, and D. Kip, Observation of parity-time symmetry in optics, Nat. Phys. 6, 192 (2010).

[194] A. Guo, G. J. Salamo, D. Duchesne, R. Morandotti, M. Volatier-Ravat, V. Aimez, G. A. Siviloglou, and D. N. Christodoulides, Observation of PT-Symmetry Breaking in Complex Optical Potentials, Phys. Rev. Lett. 103, 093902 (2009).

[195] Z. Lin, H. Ramezani, T. Eichelkraut, T. Kottos, H. Cao, and D. N. Christodoulides, Unidirectional Invisibility Induced by PT-Symmetric Periodic Structures, Phys. Rev. Lett. 106, 213901 (2011).

[196] A. Regensburger, C. Bersch, M.-A. Miri, G. Onishchukov, D. N. Christodoulides, and U. Peschel, Parity-time synthetic photonic lattices, Nature (London) 488, 167 (2012).

[197] R. Fleury, D. Sounas, and A. Alù, An invisible acoustic sensor based on parity-time symmetry, Nat. Commun. 6, 5905 (2015). 